Mme Marie-Angèle Hermitte

Mme Isabelle Doussan

M. Sébastien Mabile

Mme Sandrine Maljean-Dubois

Mme Christine Noiville

Mme Florence Bellivier

\title{
La convention sur la diversité biologique a quinze ans
}

In: Annuaire français de droit international, volume 52, 2006. pp. 351-390.

Citer ce document / Cite this document :

Hermitte Marie-Angèle, Doussan Isabelle, Mabile Sébastien, Maljean-Dubois Sandrine, Noiville Christine, Bellivier Florence. La convention sur la diversité biologique a quinze ans. In: Annuaire français de droit international, volume 52, 2006. pp. 351-390.

http://www.persee.fr/web/revues/home/prescript/article/afdi_0066-3085_2006_num_52_1_3935 


\title{
ESPACES ET COMMUNICATIONS RESSOURCES ET ENVIRONNEMENT
}

\section{LA CONVENTION SUR LA DIVERSITÉ BIOLOGIQUE A QUINZE ANS}

\author{
MARIE-ANGÈLE HERMITTE (coord.)
}

\section{SABELLE DOUSSAN, SÉBASTIEN MABILE, SANDRINE MALJEAN-DUBOIS, CHRISTINE NOIVILLE et FLORENCE BELLIVIER}

Signée à Rio en 1992 dans un contexte d'expansion du concept de développement durable ", la convention sur la diversité biologique (CDB) a quinze ans. C'est l'âge d'un premier bilan, bien difficile à effectuer tant le déploiement du texte originaire paraît immense et peu cohérent, ce qui tient à deux raisons principales : convention-cadre sans obligation juridique qu'il soit imaginable de sanctionner, la CDB requiert des instruments complémentaires pour avoir un contenu précis et obligatoire : en conséquence, les États parties ont lancé, de conférences des parties (COP) en réunions de groupes de travail, une négociation permanente visant de multiples domaines dont l'avancement est très inégal '. D'autre part, si l'on prenait au sérieux son objet, la diversité biologique,

(*) Marie-Angèle HERMITTE, directeur de recherches au CNRS - UMR 8103, pour la coordination de l'article, la première partie et les droits intellectuels des peuples autochtones; Isabelle DOUSSAN, chargée de recherches à l'INRA pour la biodiversité agricole ; Sébastien MABILE, consultant pour la biodiversité marine ; Sandrine MALJEAN-DUBOIS, chargée de recherches au CNRS - UMR 6201 pour le protocole de Carthagène; Christine NOIVILLE, directeur de recherches au CNRS - UMR 8103 et Florence BFILIVIER, professeur à l'Université de Paris X-Nanterre, UMR 8103 pour les contrats de bioprospection. L'article a été réalisé collectivement dans le cadre du réseau sur le droit des sciences et des techniques |http://www.reseaudroitsciencesettechniques.org/l. C. NoIVILLE et F. BELLIVIER remercient la revue des contrats d'avoir accepté une republication partielle du texte publié au n² 2-2007; S. MALJEAN-DUBOIS remercie les éditions du CNRS d'avoir accepté cette publication qui actualise une partie de l'article " La biodiversité dans les négociations internationales : de la Convention de Rio sur la diversité biologique au Protocole de Carthagène sur la biosécurité ", in Less biodiversités. Objets, théories, pratiques, P. MARTY/F-D. VIVIEN/J. LEPART/R. LARRËRE (dir.), CNRS éditions, 2005, pp. 21 1-226.

1. S. MALjEAN-DUBOIS: “La biodiversité dans les négociations internationales : de la Convention de Rio sur la diversité biologique au Protocole de Carthagène sur la biosécurité ", in Lés biodiversités. Objets, théories, pratiques, op. cit. p. 218. 
il faudrait en principe lui soumettre l'ensemble des activités humaines dans tous les milieux physiques connus.

L'universalité du champ d'application de la convention a débouché sur de nombreux points de friction avec d'autres instruments juridiques. Tard venue dans la construction progressive du droit de l'environnement, elle aurait dû en toute logique intégrer en les supprimant de nombreux textes assurant de manière disjointe des protections de milieux, d'habitats ou d'espèces, textes qui ne sont que des applications particulières des objectifs très généraux qu'elle poursuit. D'autres frictions viennent de sa tonalité libérale : si la CDB cherche à "protéger ", comme tout le droit de l'environnement, elle fait partie de ces textes qui utilisent des instruments économiques pour le faire et, ce faisant, se heurtent inéluctablement aux citadelles solides du droit économique, l'OMC ou l'OMPI par exemple ${ }^{2}$. Après avoir tracé les grandes lignes du texte originaire et du cadre institutionnel élaboré pour le déployer (I), nous avons choisi de délaisser certains "programmes" et certaines "questions intersectorielles " pour nous concentrer sur quatre points qui ont fait l'objet de négociations particulièrement intenses (II) : la biodiversité agricole, la biodiversité marine, le protocole de Carthagène sur les mouvements transfrontières d'organismes vivants modifiés, et les questions posées par les bioprospections, dont les droits intellectuels des peuples autochtones. La perte continue de biodiversité dans tous les milieux oblige à faire un constat un peu décourageant: qu'il s'agisse des secteurs dans lesquels des textes, facultatifs ou obligatoires, ont abouti à des compromis intéressants, ou des secteurs dans lesquels les résultats se réduisent à des intentions, l'efficacité de cette grande machinerie au regard des objectifs poursuivis reste à construire.

\section{I. - LES CARACTÉRISTIQUES DU DÉPLOIEMENT DE LA CONVENTION SUR LA DIVERSITÉ BIOLOGIQUE}

La convention sur la diversité biologique réunit aujourd'hui cent quatrevingt-dix États. Son texte est caractérisé par des objectifs d'une ambition illimitée, qui n'avaient débouché sur aucune obligation juridique que l'on puisse imaginer voir sanctionnée par une quelconque juridiction. La nature et le dynamisme des institutions mises en place étaient donc les seuls garants de la possibilité de donner un jour un contenu précis et obligatoire à ces objectifs (A). Le premier résultat de la mécanique de la convention fut l'élaboration de programmes de travail et de thématiques structurant les objectifs pour leur donner un contenu organisé sur le plan scientifique, donc accessible à l'action, qu'elle soit de nature politique ou juridique (B)

\section{A. Un modèle pur de convention-cadre}

La convention sur la diversité biologique est hardie sur le plan scientifique, s'appuyant sur un concept intégrateur permettant en principe d'englober l'ensemble du vivant dans ses relations avec les éléments abiotiques qui lui sont nécessaires (1). Si la préservation d'un certain niveau de diversité biologique avait été effectivement prise comme une contrainte minimale s'imposant à tous

2. S. Malojean-Dubois (dir.) : L'ouilil économique en droit international et européen de l'environnement, Paris, la Documentation française, 2002. 
les secteurs de l'activité humaine, elle aurait dû aboutir à une réorganisation de l'ensemble du droit international : du droit de l'environnement dont la plus grande part aurait dû devenir simplement un compartiment de la convention (tout le droit des espèces, des habitats, des zones protégées et de la qualité des milieux au minimum) ; mais aussi du reste de l'ordre juridique, dont les éléments auraient dû être revus au filtre de la préservation de la diversité biologique. Or il n'en a rien été. Bien peu de textes à ce jour ont été modifiés pour les rendre compatibles avec cet objectif. En ce sens, l'ambition scientifique n'a pas d'écho politique. C'est donc du fonctionnement des institutions (2) que l'on attend la concrétisation progressive des objectifs.

\section{Les objectifs de la convention}

Tout est parti du constat de l'insuffisance des instruments juridiques mis en place pour combattre l'extinction rapide des espèces liée à l'expansion des activités humaines. La fin du XIX ${ }^{e}$ siècle vit l'invention des parcs naturels assurant indirectement la protection des espèces qui y vivaient, le début $\mathrm{du} \mathrm{xx}^{\mathrm{e}}$ tenta les premières protections d'espèces phares comme les éléphants ou "utiles" comme les oiseaux utiles à l'agriculture. L'extinction continuait, de plus en plus massive et préoccupante. On passa à la protection des habitats. Tout ceci se révélant inutile, on adopta une vision globale du monde vivant, condition d'une biosphère viable $^{3}$, consacrée par la CDB imposant le concept de diversité biologique ; il est censé englober dans la "variabilité des organismes vivants", la diversité des écosystèmes entendus comme l'unité fonctionnelle comprenant les organismes vivants et leur environnement non vivant, la diversité des espèces ainsi que la diversité génétique au sein d'une même espèce (article 2). Le texte embrasse tous les milieux - la mer, la terre, les eaux douces - et les États se sont donné comme objectif de stopper l'extinction en 2010 quand elle semble au contraire s'accélérer. Le bilan actuel laisse perplexe tant est grande la disparité entre l'évolution encourageante des textes et la dégradation continue de la planète ${ }^{4}$.

L'article $1^{\text {er }}$ de la CDB définit trois objectifs : assurer la conservation de la diversité biologique, l'utilisation durable de ses éléments et le partage équitable des avantages résultant de l'exploitation des ressources génétiques; autrement dit, conserver grâce à l'utilité économique des ressources ${ }^{5}$ et dans le souci d'équité Nord/Sud qui caractérise le sommet de Rio. En considérant tout le spectre du vivant, dans tous les milieux et en liaison avec tous les éléments qui l'influencent, la définition donnée par la convention lui confère un champ d'action illimité, puisque tout acte humain a une conséquence sur la diversité biologique : poussé à ses limites extrêmes, le projet aurait impliqué une planification mondiale totale. Cela fut écarté par l'article 3 qui réaffirme en faveur des États

3. Le constat de l'échec des strates législatives précédentes fut au coeur de la préparation de la convention, $f$. M-A. HERMTTE: "Le concept de diversité biologique et la création d'un statut de la nature ", in B. EIPIIMAN/M-A. HERMITTE (dir), Lhomme, la nature et le droit, Bourgois, 1988, p. 238 , plus particulièrement pp. 145 et s. Voy. également J.P. BruRIER, " Le droit de la biodiversité ", $R . J E$, 1996, n $1-2$, p. 5 .

4. Cf. le triste bilan dressé par S. MaLfFAN-DLBOIs in " Environnement, développement durable et droit international - De Rio à Johannesburg et au-delà?", cet Annuaire, 2002 p. 592 ; également la communication de la Commission "Enrayer la diminution de la biodiversité à l'horizon 2010 et au-dela. Préserver les services écosystémiques pour le bien-être humain "COM (2006) 216 final.

5. Cette conception s'est imposée au cours des négociations, malgré l'affirmation de la valeur intrinsèque de la biodiversité qui n'est qu'une trace du texte initial conçu par l'UICN ; elle repose sur une idéologie de relatif bon sens - on a intérêt à protéger ce qui fonde son activité économique - mais très peu d'éléments concrets permettent de le démontrer, alors qu'il existe de nombreux exemples en sens contraire d'épuisement de ressources par surexploitation. 
"le droit souverain d'exploiter leurs propres ressources selon leur politique d'environnement".

Retenons quatre obligations acceptées par les États ; si elles sont essentielles, on voit mal comment obtenir le respect de leur exécution :

1. Les plus importantes sont définies aux articles 10 et 14 . Il s'agit d'intégrer les considérations relatives à la diversité biologique dans le processus décisionnel national et d'effectuer des études d'impact de tout ce qui est susceptible de nuire à la diversité ; l'article 7 vise l'identification des éléments constitutifs de la diversité biologique et leur surveillance, qui sont nécessaires pour mettre en œuvre les études d'impact. Il faudrait donc en principe soumettre toute décision politique à une évaluation de ses conséquences sur la diversité biologique ${ }^{6}$. Or, si l'affirmation du caractère non viable des modes de production et de consommation actuels se généralise, les seules transformations envisagées sont des retouches marginales : comme le dit le CES de l'Union européenne, la diminution de la biodiversité est l'occasion de constater une distance désolante entre le discours politique et la pratique ${ }^{7}$.

2. La seconde obligation porte sur la conservation in situ par le recours à un système de zones protégées (écosystèmes, habitats et espèces concernées, article 8) ; elle aurait dû aboutir au rattachement et à la disparition d'instruments plus anciens : convention de Ramsar, programme MAB et convention sur le patrimoine mondial de l'UNESCO... Elle est complétée par une conservation $e x$ situ dans les hypothèses les plus dégradées (article 9).

3. Chaque État doit encore élaborer des stratégies, plans et programmes nationaux assurant une politique de la diversité biologique (article 6); cela avance assez bien mais le contenu, librement décidé par l'Etat, est variable.

4. Vient ensuite tout ce qui a trait à l'accès, l'utilisation, le partage des avantages résultant de l'utilisation de la diversité biologique (articles $8 \mathrm{j}, 15,16,19$ ). Cette recherche de justice dans les échanges internationaux, d'une importance limitée pour la conservation, était essentielle pour assurer la participation des pays du Sud accusant le Nord de piller leurs ressources biologiques.

Un tel programme est hors de portée d'un texte unique. L'enjeu était donc de disposer d'institutions capables de lui donner du contenu.

\section{Les institutions appelées à déployer la convention en lui donnant progressivement du contenu}

Il est bien évident que le secrétariat de la convention, en jouant son rôle technique de rassemblement et synthèse des données, inspire de nombreuses décisions, et que la conférence des parties assume cette fonction quasi-parlementaire essentielle aux conventions-cadres. Les COP ont été réunies à des rythmes réguliers et rapides - huit conférences à ce jour - produisant de très nombreuses décisions, dont certaines ont un contenu concret important. Chaque COP est l'occasion de longues réflexions sur les différents programmes de travail. Les positions des

6. Cf. M-A. HeRMiTTE, op. cit.pp. 269 et s.

7. Le sommet de Johannesburg l'a répété sans rien changer comme le fait remarquer S. MALJEANDubols, op. cit., p. 594 ; sur ce point, $c f$. M-A. HERMITTE, "La fondation juridique d'une société des sciences et des techniques par les crises et les risques ", in C. BURTON-JEANGROS et al. : Face au risque, L'Équinoxe, Georg, 2007 p. 29 et l'exemple de la charte française de l'environnement. $C f$. aussi l'avis très sévère du CES européen sur la communication de la Commission sur la diversité biologique, JOUE C97/6 du 28 avril 2007 : la “ disparition des espèces est le résultat de millions de décisions réfléchies [...। dans le cadre de la législation existante "; autrement dit, leur disparition est le résultat de la loi. 
États, des opérateurs, des $\mathrm{ONG}^{8}$ peuvent être suivies à travers des centaines de "documents" disponibles sur le site de la convention. Les expériences des différents États sont centralisées, ce qui produit des effets de mimétisme dans la mise en œuvre par les législations nationales ou par les politiques contractuelles (centre d'échanges et d'informations).

Le bilan juridique est important. On compte au premier chef le protocole de Carthagène sur les mouvements transfrontières d'organismes vivants modifiés, texte obligatoire pour les États signataires et, à un stade moins avancé, des lignes directrices non obligatoires qui produisent cependant des effets relativement importants. Les nombreuses décisions ont en revanche un contenu généralement flou.

Mais la construction institutionnelle ne s'arrête pas là. Comme dans toutes les conventions ayant un fort contenu scientifique, des organes essentiels sont de nature scientifique. Il s'agit en premier lieu de "l'organe subsidiaire chargé de fournir des avis scientifiques, techniques et technologiques ", institué par l'article 25 . Il s'est réuni déjà onze fois et produit études et recommandations. Il a été très important dans la structuration des programmes de travail et de la hiérarchie des priorités. Il est accompagné dans sa tâche par les "groupes spéciaux d'experts techniques".

Enfin, des "groupes de travail " thématiques ont été constitués sur des points jugés essentiels, ce choix étant de nature politique puisqu'il équivaut à avancer sur des points particuliers. On verra que la relative stabilité de leur composition et la pérennité du processus de négociation ont permis, sur des sujets hautement conflictuels, d'avancer assez rapidement vers des solutions de compromis. En revanche, il faut un jour sortir du groupe pour arriver à une décision assumée par la COP. Là, le consensus se lézarde, ce qui explique la multiplication de textes sans force obligatoire. Toujours est-il que cette technique de négociation permet d'avancer, jusqu'à un certain point ${ }^{9}$.

\section{B. Le programme de travail et les thématiques mises en place}

Protéger la " diversité biologique " n'a pas de sens, car un type unique d'action ne peut convenir au regard de la diversité des écosystèmes. Or le texte de la convention évoquait sans les distinguer les écosystèmes terrestres, marins et autres écosystèmes aquatiques, et s'adressait aux espèces sauvages et domestiquées (article 2), autrement dit tout le vivant. Pour concrétiser le projet, des thèmes de travail furent dégagés par types de milieux appelant des stratégies différenciées : diversité marine et côtière, forêts, îles, eaux intérieures, terres arides et subhumides, montagnes, auxquels il faudrait ajouter les aires protégées.

La diversité biologique agricole constitue un thème de travail à part, qui concerne tous les milieux et devrait, en fait, être située dans les "questions intersectorielles". Celles-ci regroupent des thèmes divers : rapports entre diversité biologique et certaines activités humaines (tourisme, changement climatique, économie et commerce). On trouve aussi des thèmes de nature juridique (études d'impact, responsabilité et réparation, accès aux ressources génétiques et partage

8. L'influence des ONG est loin d'être négligeable, car elles interviennent directement dans l'élaboration des textes, $c f$ par exemple les lignes directrices établies comme guides pour les études d'impact, rédigées avec la participation officielle de :'International Association for Impact Assessment et la Netherlands Commission for Encironmental Assessment

9. Groupes de travail sur l'article $8 \mathrm{j}$, le partage des avantages, les aires protégées, la mise en ouvre, les mécanismes financiers. 
des avantages, connaissances traditionnelles, transferts de technologie). Enfin, sont abordées des questions techniques : conservation des plantes, initiative taxonomique mondiale (nécessaire pour savoir ce qui existe), indicateurs de biodiversité. Un problème particulier est isolé, celui des espèces exotiques envahissantes.

$\approx$

La CDB devait organiser ses liens avec les autres conventions internationales, que son article 22 prévoit en principe de respecter aussi bien que la mise en œuvre nationale de ses stratégies; elle le fait en établissant des liens avec les grandes régions ou sous-régions du monde (qui permettent des parcs naturels transfrontières par exemple) et en travaillant avec les " points focaux " nationaux. C'est donc une vaste toile qui a été tissée, de manière transversale au niveau international, de manière verticale en direction des États. Il s'agit d'une construction lente et molle caractéristique de ces grandes conventions-cadres ${ }^{10}$. Ces défauts apparents sont la condition pour que des régions du monde aux intérêts divergents puissent aller à la négociation et rapprocher leurs points de vue et leurs urgences. À cet égard, le bilan de la convention est plutôt satisfaisant, le mécanisme ayant réussi à attirer dans des opérations de conservation des régions du monde pour lesquelles ce n'était pas une priorité. Pourtant, l'absence de contrôles crédibles de la mise en œuvre et les difficultés que rencontre la convention à lutter contre les intérêts économiques présents dans d'autres instruments internationaux conduit à douter de son efficacité ${ }^{11}$.

\section{II. - UN DÉPLOIEMENT PEU COHÉRENT ET D'UNE EFFICACITÉ LIMITÉE}

La manière dont la CDB réalise ses objectifs laisse perplexe. Des points essentiels se heurtent à de graves et anciennes difficultés connues de tous, en raison d'une volonté politique défaillante (A). D'autres, médiatiques mais plutôt périphériques, ont avancé très vite, comme la question du risque biotechnologique ou de la bioprospection (B). On pourrait encore affirmer que l'avancement assez satisfaisant des aires protégées est la conséquence d'un mouvement de pensée bien antérieur à la convention qui semble avoir des difficultés à hiérarchiser les urgences ${ }^{12}$.

\section{A. Des objectifs importants qui se heurtent à d'autres instruments internationaux}

Nous choisirons deux exemples. Le premier concerne la diversité biologique qui entretient des rapports avec l'agriculture (1). Le second porte sur la biodiversité marine et la création de parcs marins (2). Ils appellent l'un comme l'autre des

10. S. Maljean-Dubois : “ Environnement, développement durable et droit international - De Rio à Johannesburg et au-delà ?", cet Annuaire, op. cit (voy. supra).

11. S. Mal.JEAN-Dubois : "La biodiversité dans les négociations internationales $|\ldots|$ ", op. cit., p. 217

$12.11 \%$ des terres mondiales sont plus ou moins protégées, comprenant le coeur de zone, souvent une zone périphérique et, beaucoup plus rarement cet élément essentiel que sont les corridors qui permettent de recréer une relative continuité entre les zones; ce n'est qu'à la septième COP, que la CDB s'est dotée d'un programme de travail sur les aires protégées, décision VII/28. 
actions urgentes, mais peinent à dégager des solutions obligatoires tant ils se heurtent à la puissance d'instruments juridiques préexistants représentant des intérêts économiques que les autorités publiques ne se décident pas à soumettre à la contrainte écologique, allant de compromis en compromis.

\section{L'exemple de la biodiversité agricole}

L'agriculture occupe une proportion importante des terres. Lors du défrichement, la perte de biodiversité sauvage est massive. Ensuite, se constitue une autre biodiversité, agricole ${ }^{13}$. Dans ce deuxième contexte, l'agriculture moderne réduit sans cesse la biodiversité cultivée, par sa concentration sur un petit nombre d'espèces et de variétés. L'utilisation de molécules chimiques réduit ce qui reste de biodiversité sauvage, avec des inquiétudes particulières pour les insectes pollinisateurs ${ }^{14}$, et la dégradation générale des écosystèmes diminue la fertilité des terres. Il était donc logique que la convention compte ce secteur dans son champ (articles 2 et 10 ). Mais elle le fait sans autre précision ${ }^{15}$, et c'est dans les travaux de la COP que la dimension internationale du traitement des rapports entre diversité biologique et agriculture doit être recherchée (1.1). La mise en œuvre de la convention passe par l'obligation d'intégration de ses objectifs dans les autres politiques, dont la politique agricole. Si l'on prend l'exemple de la politique agricole commune menée par l'UE, on constate une importante érosion des ambitions (1.2). Attachée à son objectif de compétitivité internationale, l'UE conçoit ses stratégies dans l'optique de l'OMC, ce qui pérennise les défauts connus du système de production agricole.

\subsection{La COP, l'activité agricole et la diversité biologique : de vastes ambitions}

Se déchargeant sur la FAO d'un certain nombre de tâches comme la conservation ex situ des ressources génétiques domestiques et la protection des végétaux ${ }^{16}$, la $\mathrm{CDB}$ a développé une vision critique des pratiques agronomiques conventionnelles, mais elle n'a pas les moyens de la mettre en œuvre.

13. La décision V/5 définit la diversité biologique agricole : il s'agit de tous les éléments constitutifs de la diversité biologique qui relèvent de l'alimentazion et de l'agriculture et de toutes les utilités qu'elle présente : réservoir de ressources génétiques, fourniture de services écologiques comme la régulation des parasites, le maintien du cycle hydrologique, la régulation du climat.

14. La CDB lui consacre un programme de travail particulier, mais depuis l'international pollinisators initiative, qui fit l'objet d'une déclaration à Sao Paulo en 1999 (http://www.biodiv.org/doc/ref/agrpollinator-rpt.pdf), les choses n'ont pas beaucoup avancé.

15. Sur la quasi-absence de prise en compte des rapports entre l'agriculture et la biodiversité dans l'ensemble du droit international de l'environnement, lire S. MALJFAN-DuBOIS, "PAC et conservation de la biodiversité : un enjeu non traité par le droit international de l'environnement ", pp. 315 et s. in "Conservation de la biodiversité et PAC, des mesures agro-environnementales à la conditionnalité environnementale ", sous la direction de I. DousSAN et J. DUBOIS, Paris, la Documentation Française, 2007.

16. Elle le fit par la décision II/5, et la question est désormais réglée par le traité de 2001 sur les ressources phytogénétiques, qui ne repose pourtant pas sur les mémes principes juridiques en matière de souveraineté, accés et échange, $c f$. C. NoIvil.LE: "Le statut juridique des collections de ressources génétiques ", in M.-A. HrkMITTE (dir.), Les Ressources génétiqués iégétales et le Droit dans les rapports nord-sud, Bruylant, Bruxelles, 2004, pp. 219 et s. et. A. SoNTot : Processus internationaux de création de normes dans un sous-système complexe' le cas de la génétique végétale pour l'agriculturé é l'alime'nta. tion, Thèse IEP Paris sous la dir. de M-C. Smouts, décembre 2006 ; Bonaventure Dossot:-Yovo : Lacies aux ressources biologiques dans les rapports Nord-Sud : Enjeux, jeux et perspectices de la protection des satoirs autochtones, Thèse Liège sous la dir. de M. Mormont et I. Stengers, déc embre 2006. 


\section{a. La COP et sa vision critique des pratiques agricoles conventionnelles}

L'approche de la COP est exposée très clairement en annexe de la décision III/11 "relative à la conservation et l'utilisation durable de la diversité biologique agricole ". Après avoir rappelé l'importance de cette biodiversité pour la sécurité alimentaire et sa compétence en la matière, elle explique comment l'agriculture dépend de la diversité biologique, particulièrement des micro-organismes du sol, des insectes pollinisateurs, des espèces susceptibles d'être cultivées ou élevées, et comment elle produit en retour des effets sur l'environnement. Les pratiques agricoles conventionnelles consommatrices d'intrants ne seraient pas "viables " du fait de la monoculture, de la mécanisation excessive et des abus de produits chimiques. Les incidences socio-économiques ne sont pas oubliées, particulièrement l'uniformisation des cultures des communautés traditionnelles. En revanche, d'autres pratiques agricoles, tant traditionnclles que modernes, sont identifiées comme favorables à la diversité biologique.

\section{b. Un programme de travail plus que des réalisations concrètes}

Dès 1996, la COP dressa une liste des domaines d'activité importants pour la diversité, retenant deux domaines prioritaires, la disparition progressive des pollinisateurs et celle des micro-organismes des sols ${ }^{17}$. Quatre ans plus tard, le programme fut officiellement lancé par une décision voulant favoriser les effets positifs et lutter contre les effets négatifs des pratiques agricoles. Il se caractérise de la manière suivante : une approche par écosystèmes ; une approche multidisciplinaire prenant en compte les aspects scientifiques, sociaux et économiques ; une approche fondée sur la connaissance de l'état et de l'évolution de la diversité biologique agricole pour, après études de cas, recenser les méthodes, techniques, politiques de gestion, mécanismes incitatifs qui favorisent la diversité biologique ou atténuent sa dégradation, et qui accroissent sa capacité à satisfaire les besoins, ce qui est qualifié de gestion " souple " ou " adaptative "; un " renforcement des capacités " des agriculteurs, des communautés autochtones et locales et de leurs organisations pour donner une conscience plus aiguë de la nécessité de bien gérer la diversité agricole. Le suivi de ce programme fait l'objet d'observations diverses ; un calendrier allant de 2003 à 2007 a été fixé, qui devrait permettre de dresser un bilan des actions entreprises par les États.

L'approche et le programme de travail sont intéressants, mais les réalisations passent par des politiques nationales ou régionales. Or, en Europe, les autorités communautaires sont beaucoup moins critiques à l'égard des pratiques existantes. Si, à l'échelle communautaire, le constat de la dégradation de la biodiversité est bien dressé, il n'est pas imputé au mode de production lui-même mais à un manque de maîtrise des processus biotechniques, à des lacunes de la réglementation environnementale, au laxisme des agriculteurs et des autorités

17. Ressources en eau, ressources génétiques des végétaux et des animaux, intrants, savoirs traditionnels, marchés agricoles. COP V : création de l'“ initiative internationale pour la conservation et l'utilisation durable des pollinisateurs ". COP VI : création de l'" initiative intersectorielle sur la diversité biologique des sols". La CDB accorde également une grande importance aux " technologies de réduction de l'utilisation des ressources génétiques "(GURT), qui empêchent la germination d'une graine après la récolte (Terminator) ou qui empêchent une plante d'exprimer un caractère bénéfique, par exemple une résistance à la sécheresse, si une pulvérisation spécifique ne lui a pas été faite. Dans les deux cas, il s'agit d'obliger les agriculteurs à racheter leur semence chaque année. Ces technologies ont fait l'objet d'un moratoire (décision V/5 III en 2000), prolongé lors de la COP VIII à la demande de certaines ONG ("Interdire Terminator") et du Parlement européen (Résolution du 16 mars 2006) P6_TA(2006)0098 ; pour une position différente, $c f$. le point de vue de l'Union internationale pour la protection des obtentions végétales concernant la décision VI/5 de la COP à la CDB, adoptée par le Conseil le 11 avril 2003. 
publiques dans l'application de règles protectrices des milieux naturels; il est admis que tout cela pourrait être combattu à la marge, par "l'agriculture raisonnée " par exemple ${ }^{18}$. Moins critique donc, l'Union européenne voit ses volontés de réforme sévèrement encadrées par les contraintes de l’OMC.

\subsection{La mise en suvre de la CDB contrariée par le droit communautaire}

Pourtant, la mise en œuvre de la CDB par l'UE revêt une importance particulière : l'agriculture occupe près de la moitié d'un territoire qui abrite une grande diversité d'habitats naturels. Il était donc logique que la stratégie communautaire en faveur de la diversité biologique élaborée par la Commission en février 1998 et présentée comme la mise en œuvre des engagements de l'UE dans le cadre de la convention sur la diversité biologique, débouchât sur des « plans d'action ", dont l'un était consacré à l'agriculture ${ }^{19}$. Mais à ce stade, la référence à la CDB s'est faite moins présente, son influence étant contrecarrée par d'autres sources du droit d'inspiration différente.

On observe, certes, des normes issues du droit de l'environnement (encadrement de la pollution des eaux par les nitrates d'origine agricole ou de la dissémination volontaire d'OGM). Le cinquième programme d'action en matière d'environnement, de 1992 à 2000, identifiait l'agriculture comme un "secteur cible ", notant qu'une réforme de la $\mathrm{PAC}$ était indispensable. Le sixième programme communautaire d'action pour l'environnement concerne les forêts, les sols, les OGM. Mais une inspiration différente vient de la PAC dont nombre d'observateurs font la cause principale de l'état de la diversité biologique agricole ${ }^{20}$, ce qui est d'ailleurs officiellement admis par la Commission ${ }^{21}$, qui attribue à la PAC une grande partie de la responsabilité de l'intensification de l'agriculture, et particulièrement de l'utilisation accrue d'engrais et de pesticides. Mais, alors que la COP dénonçait "l'utilisation inappropriée " des intrants chimiques et le caractère " non viable " du modèle qui en résultait, la Commission insiste sur les contraintes qui conduisent à pérenniser le modèle d'une " agriculture plus performante et répondant mieux aux attentes du consommateur. Dans le contexte international, il faut que l'Europe soit en mesure de tirer parti de l'augmentation prévisible de la demande mondiale pour bon nombre de produits tels que les céréales, la viande bovine et les produits laitiers à valeur ajoutée " 22 . Certes, "les considérations environnementales [...] sont une composante essentielle des propositions de la Commission " mais il s'agit “ d'améliorer l'équilibre entre l'agriculture intensive et l'environnement, de manière à éliminer des éléments nuisibles de l'agriculture intensive et à améliorer l'image de l'agriculture en la faisant apparaître comme un secteur vivant en harmonie avec son milieu [...] le message principal des propositions de réforme de la politique agricole commune dans le cadre d'Agenda 2000 est l'accroissement de la compétitivité. Il n'entre pas dans les intentions de la Commission de saper les positions concurrentielles des agriculteurs en accumulant les exigences environnementales au-delà du raisonnable " 23 . Le traitement de la

18. I. DousSAN, “ Entre contrainte et incitation : analyse juridique de la qualification au titre de l'agriculture raisonnée ", INRA, Sciences Sociales, $n^{3} 3,2004$.

19. Communication de la Commission du 27 janvier 1999, "Pistes pour une agriculture durable ", COM(1998) 42 ; Plan d'action en faveur de la diversité biologique dans le domaine de l'agriculture, COM(2001) 162 final.

20. N. de SAADELER/C.H. BORN (dir) : "Droit international et communautaire de la biodiversité ", Dalloz, 2004, 624.

21. COM(1999) 22 final.

22. Ibid.

23. Ibid. 
biodiversité agricole est donc en fait totalement dépendant des mesures de soutien public à l'agriculture, la question se posant alors de savoir si les mesures de la PAC favorables à la diversité biologique (a) ne sont pas essentiellement conçues au regard de la stratégie de l'UE à l'OMC (b) ${ }^{24}$.

\section{a. La biodiversité dans les régimes de soutien de la PAC}

Dans le cadre de la PAC, il n'existe pas spécifiquement de mesures relatives à la biodiversité ; l'environnement apparaît dès 1992 dans le règlement 2078 concernant " des méthodes de production agricole compatibles avec les exigences de la protection de l'environnement ainsi que l'entretien de l'espace naturel ", et le noyau dur du dispositif relatif à la biodiversité agricole sera inséré dans le deuxième pilier, "développement rural" 25 . Il introduit des mesures agroenvironnementales (MAE) qui incluent plusieurs options favorables à la diversité biologique telles que l'agriculture biologique, les méthodes de culture à faibles consommations intermédiaires, l'entretien écologique des terres agricoles abandonnées, l'élevage de races animales menacées ou la culture de variétés traditionnelles locales 26 .

Sont également favorables à la diversité biologique les soutiens venant compenser des inconvénients : difficultés naturelles des régions montagneuses ou difficultés liées aux contraintes des zones soumises à des exigences environnementales particulières, comme le réseau Natura 2000. D'autres soutiens peuvent être apportés sous forme d'aides à la formation ${ }^{27}$, à la préretraite ${ }^{28}$, à la sylviculture ou encore à la mise sur le marché de produits de qualité ${ }^{29}$. La contrainte environnementale a encore été intégrée au " premier pilier " de la PAC, la politique des marchés. Il s'agit par exemple des mesures de "gel des terres " destinées à lutter contre la surproduction, des incitations à l'"extensification " de l'élevage des races bovines destinées à la boucherie.

En revanche, ce qui pose problème est la "conditionnalité " des nouveaux soutiens aux agriculteurs, intitulés “droits à paiement unique »30. En principe, le versement de l'aide est conditionné au respect des « exigences réglementaires » du

24. "L’idée générale [...] est que les agriculteurs devraient assurer un minimum d'activités en faveur de l'environnement en tant que partie intégrante des régimes de soutien, mais que toute prestation environnementale supplémentaire au-delà du niveau de base des bonnes pratiques agricoles et du respect de la législation en matière de protection de l'environnement devrait être rémunérée par la société au moyen des programmes agro-environnementaux ", Communication de la Commission du 27 janvier 1999, "Pistes pour une agriculture durable ", COM(1999) 22 final.

25. Le règlement 1257 du 17 mai 1999 a été le premier texte relatif au développement rural ; il a été abrogé par le règlement 1698 du 20 septembre 2005 concernant le soutien au développement rural par le Fonds européen agricole pour le développement rural (Feader).

26. Les MAE prévoient l'indemnisation des agriculteurs qui s'engagent, sur une base volontaire à fournir un service environnemental sur une période de cinq ans. Le montant dépend des coûts encourus et des pertes de revenu subies et ne sera accordé qu'en contrepartie de mesures qui vont au-delà de la simple application des bonnes pratiques agricoles habituelles. Celles-ci correspondent aux principes agricoles qu'un agriculteur raisonnable appliquerait dans la région concernée et que les États membres définissent par des standards dans leurs plans de développement rural.

27. Connaissance des écosystèmes, plans de gestion de la faune et de la flore, etc.

28. Réaffectation des terres libérées pour la protection d'écosystèmes.

29. " En limitant l'utilisation de certaines dénominations à un nombre restreint de produits élaborés à l'aide de ressources locales et traditionnelles, les mesures relatives à la qualité contribuent en effet à préserver la diversité biologique. "Plan d'action en faveur de la diversité biologique dans le domaine de l'agriculture, COM(2001) 162 final.

30. Intitulé regroupant les anciennes aides agricoles. Ces DPU sont désormais “découplés " de la production ; cela signifie que leur montant a été calculé sur la moyenne des aides perçues les années précédentes et qu'ils sont désormais dus quelles que soient les quantités produites ou les surfaces exploitées par l'agriculteur, règlement 1782 du 29 septembre 2003, JOUE L 270/1 du 21 octobre 2003. 
droit communautaire et des " bonnes conditions agricoles et environnementales " définies par les États membres, ce qui pourrait en faire un outil puissant du changement de modèle agronomique. Mais le système a été conçu sur une base historique d'attribution des droits qui l'éloigne de son objectif environnemental ${ }^{31}$. En conséquence, "l'éco-conditionnalité peut limiter les effets environnementaux pervers des aides, mais n'assure pas que les aides soient versées là où elles engendreront la plus grande production de biens environnementaux. Même avec une éco-conditionnalité renforcée, il n'y a pas de raison que la biodiversité ou les paysages produits par les agriculteurs soient proportionnels à la surface qu'ils exploitent" 32 . Pour que ces soutiens contribuent à renforcer les services environnementaux, une profonde réforme de leur clef de répartition serait nécessaire, et l'Union européenne n'en prend pas le chemin car la conditionnalité est un élément de sa stratégie à l'égard de l'OMC.

\section{b. La biodiversité dans la stratégie européenne de négociation à l'OMC}

Les dernières grandes réformes de la PAC, en 1999 et 2003, ont été principalement motivées par l'ouverture progressive à la concurrence des marchés agricoles internationaux dans le cadre de l'OMC. L'Union européenne y a défendu une agriculture dite "multifonctionnelle", assurant une fonction économique, sociale et environnementale ${ }^{33}$. "Reste que par la défense de ce modèle, l'Union européenne entend aussi, si ce n'est surtout, maintenir ses mesures de soutien les plus essentielles à la survie de la PAC " 34 ; il s'agit d'éviter le démantèlement trop brutal de l'interventionnisme traditionnel. La protection de l'environnement justifie les mesures de soutien public aux agriculteurs, en les faisant migrer vers la "boîte verte" afin de les faire échapper aux engagements de réduction ${ }^{35}$. $90 \%$ des soutiens internes que l'UE s'était engagée à réduire de $70 \%$ ont ainsi rejoint la boîte verte ${ }^{36}$.

Malgré des différences factuelles, on retrouve des difficultés du même ordre avec la biodiversité marine, tant les projets de protection se heurtent aux conventions internationales antérieures, aux contraintes économiques et à la nécessité d'une mise en œuvre nationale dont l'inexécution n'est pas sanctionnée.

31. J. LEPART/P. MARTY/J. TERRAUBE, "Les MAE et la biodiversité, quel bilan?" p. 149, in I. Doussan/J. DuboIS, (dir) : “Conservation de la biodiversité et PAC : des mesures agro-environnementales à la conditionnalité environnementale ", op. cit. et I. DoUsSAN, "La conditionnalité des aides agricoles : continuité ou innovation?", ibid., pp. 177 et s.

32. T. LE COTTY, "Les impacts de l'OMC sur la prise en compte de la biodiversité dans la PAC ", pp. 275 et s. in I. Dotssan/J. DuboIs (dir): "Conservation de la biodiversité et PAC': des mesures agroenvironnementales à la conditionnalité environnementale ", op. cit.

33. Sur le concept de multifonctionnalité agricole, voy. Économie rurale, n' spécial Multifonctionnalité, $n^{\circ}$ 273-274, 2003. Plus particulièrement A. MASSOT-MARTı : " Le paradigme multifonctionnel : outil et arme dans la renégociation de la PAC ", p. 30. Voy. également, D. SORIN : "La réforme de la PAC et les négociations multilatérales ", Revue de Droit rural, juin-juillet 2005, p. 14 .

34. N. RUbio/N. Thome: : Subventions agricoles en faveur de la protection de l'environnement : le droit communautaire à l'épreuve du droit de l'OMC", pp. 251 et s. in I. Doussav/J. DuBOIS (dir): . Conservation de la biodicersité et PAC: des mesures agro-encironnementales a la conditionnalite encironnementale ", op. cit.

35. Schématiquement, les subventions sont classées catégories autorisées (verte), soumises à réduction (orange) et interdites (rouge). Les soutiens versés "au titre de programmes environnementaux" sont rangés dans la "boite verte".

36. J. BERTHELoT : La boite verte : une boite noire qui cache la boite en or, cité par N. RI'BIO) et N. Thome /http://www.wto.org/french/forums_f/ngo_f/pospap_f. htm l. 


\section{L'exemple de la diversité biologique des zones marines et côtières}

La diversité biologique marine est, comme la diversité biologique agricole, d'une importance considérable au regard de son étendue - $70 \%$ de la surface terrestre - de sa part majoritaire dans la biodiversité totale et du rôle des océans dans la séquestration du carbone. Pourtant lorsque la communauté internationale adopte la convention sur la diversité biologique, celle-ci ne fait référence aux écosystèmes marins que dans la définition de la diversité biologique donnée à l'article 2. De plus, le milieu marin est déjà structuré juridiquement par des conventions internationales qui opposent des obstacles à une prise en compte de la protection de l'environnement. Dès lors, la COP devra inventer de toutes pièces un cadre juridique pour son action, en tenant compte de ces difficultés : ce sera le mandat de Jakarta (2.1). D'une mise en œuvre difficile, il est largement réalisé dans un cadre national et surtout régional comme le montre son application en Méditerranée (2.2). Enfin, on exposera la manière dont la COP apprécie les enjeux et les possibilités d'action pour l'avenir : changement climatique, statut de la haute mer, récifs coralliens (2.3).

\subsection{La COP confrontée au silence de la CDB et à l'existence d'autres instruments internationaux : le mandat de Jakarta comme cadre pour l'action}

\section{a. La CDB confrontée au contexte juridique antérieur}

L'article 22 de la CDB dispose que la convention ne modifie pas les obligations préexistantes, "sauf si l'exercice de ces droits ou le respect de ces obligations causait de sérieux dommages à la diversité biologique ", et précise que "les Parties contractantes appliquent la présente Convention, en ce qui concerne le milieu marin, conformément aux droits et obligations découlant du droit de la mer ". Or, la convention des Nations Unies sur le droit de la mer (CNUDM), adoptée en 1982, a été élaborée conformément à la pensée politique des années 1970, ce qui entraîne deux difficultés pour la CDB.

On pouvait regretter la pauvreté relative du droit de la mer en matière de protection de l'environnement, qui n'est abordée que de manière accessoire et sans référence au concept de biodiversité, encore inconnu à l'époque où le traité a été rédigé. Pourtant, la CNUDM avait été reçue en son temps comme une ouverture offerte au droit de l'environnement, ce dont témoigne le rapport Brundtland: "la première et la plus importante initiative que les États peuvent prendre dans l'intérêt des océans est la ratification de la CNUDM $* 37$. Cette opinion sera confirmée par l'Agenda $21^{38}$ et réaffirmée en 2002 à Johannesburg ${ }^{39}$. Effectivement, l'article $194, \S 5$, de la CNDUM fait peser sur les États une obligation générale de protection du milieu marin dans l'ensemble des espaces maritimes. Il précise que les mesures de protection de l'environnement marin doivent inévitablement comprendre celles qui sont " nécessaires pour protéger et préserver les écosystèmes rares ou fragiles ainsi que l'habitat des espèces et autres organismes marins en régression, menacés ou en voie d'extinction ". Cette disposition fournit une base juridique aux accords internationaux

37. Rapport de la commission mondiale sur l'environnement et le développement, chapitre $10, \$ 55$ (Doc. : A/RES/42/187, 11 décembre 1987); les auteurs semblaient donner une interprétation plus large à la protection de l'environnement que l'interprétation d'origine, limitée à la lutte contre la pollution.

38. A/CONF. 151/26/Rev.1 : Action 21.

39. Point 30 a) du Plan d'application du SMDD, 4 septembre 2002 (A/CONF.199/20); les États sont invités à ratifier la CNDUM. 
prévoyant, entre autres choses, l'instauration d'aires marines protégées destinées à protéger et préserver les écosystèmes et les habitats d'espèces. Tout comme l'obligation prévue à l'article 192, elle est applicable à l'ensemble des espaces maritimes, y compris la haute mer ${ }^{40}$.

En reconnaissant la CNUDM comme base légale de la protection des océans, l'Agenda 21 consacrait la souveraineté territoriale de l'État côtier, le droit d'exploiter les ressources biologiques qui en découle et l'État côtier comme garant de la protection de l'environnement, donc de la diversité biologique, - ce qui correspond trait pour trait aux principes généraux de la CDB.

Une difficulté venait pourtant de l'articulation entre les catégories juridiques de base des deux instruments. La CDB repose entièrement sur les droits souverains des États sur leurs ressources biologiques, ce qui comprend leur droit de les exploiter sclon leur propre politique d'environnement (article $3 \mathrm{CDB}$ ). La CNUDM s'en distingue. En effet, à côté de la reconnaissance de la pleine souveraineté étatique sur la mer territoriale à 12 milles des lignes de base, cohérente avec la CDB, elle a étendu les zones de juridiction nationale jusqu'à 200 milles, l'État riverain ayant alors des droits exclusifs sur certaines ressources naturelles. En compensation, l'État a "l'obligation de protéger et de préserver le milieu marin " (article 192 de la CNUDM). En revanche, la haute mer et la Zone internationale des fonds marins échappent à l'appropriation des États et conservent le principe de liberté ; celui-ci est atténué, en ce qui concerne "la Zone ", par la qualification de "patrimoine commun de l'humanité" (article 136 de la CNUDM). Or la CDB ne consacre pas de régime de liberté, qui reste donc propre à la haute mer, et a volontairement écarté la catégorie de patrimoine commun de l'humanité. Dès lors, en l'absence de souveraineté ou de juridiction nationale sur ces espaces marins, comment concilier les impératifs de protection issus de la CDB avec les dispositions de la CNUDM ? En cas de conflits de conventions, c'est le droit de la mer qui a la primauté en vertu de l'article 22.2 de la CDB et de l'article 237 de la CNUDM. L'établissement d'aires marines protégées en haute mer s'opposerait par conséquent au principe de liberté de la navigation garantie par l'article 87 de la CNUDM ${ }^{41}$, et la force coutumière de ce principe fait que le droit international de l'environnement est relativement réticent à privilégier la nécessité de protection de l'environnement sur les principes juridiques du droit de la mer ${ }^{42}$. C'est cette logique que l'on va chercher à bousculer à partir du mandat de Jakarta.

\section{b. Le mandat de Jakarta comme cadre d'action}

La thématique de la biodiversité marine et côtière avait été laissée de côté au cours des débats préparatoires de la CDB ${ }^{43}$. Le SBSTTA (organe subsidiaire

40. Cf. T. ScovazzI., " Marine Specially Protected Areas and Present International Law of the Sea ", in "Nouvelles technologies et droit de l'environnement marin", Kluwer Law International, The Hague, London, Boston, 2000, p. 186.

41. Sur l'articulation de la CDB avec le droit de la mer, voy. Ch. DE, FonTAuBERT/D. R. DOWNES/ T.S. AGARDY, Biodiversity in the Seas. Implementing the Convention on Biological Biodiversity in Marine and Coastal Habitats, IUCN Environmental Policy and Law Paper $\mathrm{n}^{\circ} 32$, Gland et Cambridge, IUCN, 1995.

42. Sur le sujet, $c f$. F. SPAII : " Navigation in Marine Protected Areas : National and International Law ", in Ocean Development \& International Law, vol. 31, 2000, pp. 285-302 et A. MERIALDI, : "Legal Restraints on Navigation in Marine Specially Protected Areas ", in T. ScovazzI (ed): "Marine Specially Protected Areas - The General Aspects and the Mediterranean Regional System ", The Hague, Kluwer Law International, 1999.

43. Cf. G. Verhoosel, " Prospecting for Marine and Coastal Biodiversity : International Law in Deep Water ", in International Journal of Marine and Coastal Law, vol. 13, n 1, mars 1998, pp. 91-104. 
chargé de fournir des avis scientifiques, techniques et technologiques) montra son utilité en prenant le relais pour poser les bases scientifiques de l'action, dans un contexte où l'incertitude scientifique est encore plus prégnante qu'ailleurs. Il y a encore peu d'années, les scientifiques pensaient que la vie n'était pas possible audelà d'une certaine profondeur en raison de l'absence de lumière ; on a, depuis, démontré qu'il existait dans les grands fonds marins des "oasis " de biodiversité, concentrant une multitude d'espèces inconnues, y compris dans les zones polaires. Même dans les zones côtières métropolitaines, la cartographie et la mise en œuvre des outils de connaissance (ZNIEFF : zone naturelle d'intérêt écologique, faunistique et floristique) sont encore incomplètes, alors que la raréfaction des ressources halieutiques dans ces zones pousse les pêcheurs à mobiliser des moyens industriels toujours plus pernicieux, toujours plus loin et plus profondément.

Le SBSTTA émit donc une première recommandation qui insistait sur l'importance de la conservation in situ sous la forme d'aires marines protégées (article 8 de la CDB) ${ }^{44}$. En 1995, la notion d'aire marine protégée était encore émergente et ne bénéficiait pas du consensus dont jouissaient les aires protégées terrestres, plus anciennes. La notion de "frontière " paraissait difficile à concevoir dans ce milieu libre et ouvert qu'est la mer. On craignait aussi que la fluidité du milieu marin ne fasse obstacle aux efforts de conservation et il fallut du temps pour prouver leurs effets positifs en termes d'augmentation de la biomasse, $y$ compris sur les espaces avoisinants. En annexe de cette première recommandation, le SBSTTA identifiait les principales thématiques du "mandat de Jakarta ".En 1995, la seconde COP adopta la décision II/10 relative à " la conservation et à l'utilisation durable de la diversité biologique marine et côtière ", appelée plus tard "mandat de Jakarta". C'est l'ébauche d'un programme de travail ${ }^{45}$ qui sera précisé en 1998 par la décision IV/5 ${ }^{46}$. Elle identifie six principes de base issus des principes généraux de la $\mathrm{CDB}$ - approche par écosystème, principe de précaution, importance de la science, rôle des experts, engagement des communautés locales et indigènes - et implique trois niveaux de mise en œuvre (national, régional et global) et cinq thématiques propres au milieu marin: gestion intégrée des zones côtières, particulièrement importante en raison de la gravité des pollutions telluriques qui représenteraient $80 \%$ de la pollution du milieu marin ; utilisation durable des ressources marines et côtières vivantes, point de friction non résolu avec la mariculture et la pêche; aires marines et côtières protégées ; mariculture ; génotypes exotiques.

Au fil des COP, l'accent fut mis sur le blanchiment des coraux et l'importance d'une organisation en réseau des aires protégées ${ }^{47}$; parce qu'elles « contribuent à préserver la diversité biologique, à garantir l'utilisation durable des ressources, à gérer les conflits et à améliorer le bien-être économique ainsi que la qualité de vie ", elles sont "un des outils essentiels pour la conservation et l'utilisation durable de la diversité biologique marine et côtière "48. Après capitalisation des travaux du SBSTTA, la décision VII/5 esquisse un "cadre de gestion des

44. Recommandation $\mathrm{V} / 8$, Aspects scientifiques, techniques et technologiques de la conservation et de l'utilisation durable de la diversité biologique marine et côtière (UNEP/CDB/COP/2/5).

45. Il prend sa place à côté de la diversité biologique agricole, des terres arides et sub-humides, des forêts, des eaux intérieures, des montagnes, et de la diversité biologique insulaire dont le programme de travail vient d'être adopté à la huitième COP en mars 2006. Son initiative repose essentiellement sur les pays océaniens.

46. Décision IV/5 sur la conservation et l'utilisation durable de la biodiversité marine et côtière.

47. $C f$. la décision VII-5 puis la décision VIU/28 sur les aires protégées qui stigmatise la sous représentativité du milieu marin au sein des aires protégées existantes.

48. Décision VII/5, $\$ \$ 12$ et 16. 
éléments de la biodiversité marine et côtière " qui accorde une place privilégiée aux aires marines protégées déclinées en deux niveaux de gestion ${ }^{49}$ : celles au sein desquelles les menaces sur l'environnement sont "gérées ", des prélèvements étant autorisés dans une perspective d'utilisation durable des ressources, et celles où les activités humaines sont strictement contrôlées, voire interdites, de manière à maintenir l'intégrité, la structure et le fonctionnement des écosystèmes. L'importance des aires protégées est reprise dans le programme adopté à Kuala Lumpur ${ }^{50}$. Il insiste sur la nécessité pour les parties de s'organiser au niveau régional pour établir effectivement ces aires, les intégrer dans un réseau global, assurer l'effectivité de leur gestion, tout en facilitant la recherche et la surveillance ainsi que le suivi de la gestion (objectifs 3.1 à 3.5 ).

Enfin, conformément aux recommandations du SBSTTA et aux conclusions du sommet de Johannesburg, la thématique des aires marines protégées est étendue vers les zones placées au-delà des limites de juridiction nationale 51 (objectif 3.2). Sur ce point particulièrement délicat au regard du statut de liberté de la haute mer, la conférence des parties s'adresse au secrétariat exécutif de la convention qui devrait soutenir le travail de l'Assemblée générale des Nations Unies pour identifier des mécanismes de création et de gestion de telles aires en haute mer, et plus particulièrement la division des affaires maritimes et du droit de la mer des Nations Unies ${ }^{52}$.

\subsection{La solution régionale d'une mise en œuvre diffícile}

Force est de constater que la mise en œuvre du mandat de Jakarta s'appuie essentiellement sur des instruments juridiques antérieurs à la CDB, qui privilégient une approche régionale pour la lutte contre les pollutions et l'établissement de zones protégées. Ces instruments reposaient déjà sur l'idée d'une unité des différents milieux, considérant l'élément liquide comme un trait d'union entre les États riverains, ce qui conduisait directement au concept de mer régionale ${ }^{53}$; le régionalisme, en favorisant la coopération entre les États, est "le meilleur moyen de gérer rationnellement un espace déterminé, tel que le sont les mers régionales " 54 . Effectivement, certaines conventions relatives à des mers régionales ont fait l'objet d'une révision afin de prendre en compte les exigences en matière de protection de la biodiversité issues de la CDB.

49. Décision VII/5, annexe I, appendice 3.

50. Décision VII/5, annexe I, §III, éléments du programme.

51. Le SBSTTA a adopté au cours de sa huitième réunion une recommandation au sein de laquelle il appelle les parties à convenir " qu'il est urgent d'établir de nouvelles zones marines et côtières protégées dans les régions ne relevant d'aucune juridiction nationale, englobant notamment des monts sousmarins, des bouches hydrothermales, ainsi que des écosystèmes coralliens d'eau froide et de haute mer, conformément au régime juridique international et en se fondant sur les données scientifiques" $18^{*}$ réunion du SBSTTA, recommandation VIV/3 B, point 19, 10-14 mars 2003 ; projets de décisions destinées à la 7* conférence des parties à la convention sur la diversité biologique, note du secrétariat général, 15 janvier 2004 , p. 53 (doc. : UNEP/CBD/COP/7/1/Add.2]).

52. La collaboration doit également s'étendre à l'Organisation maritime internationale (OMI), l'Autorité internationale des fonds marins, la FAO, les organisations régionales responsables des pêches, la Commission océanographique intergouvernementale (COI) de l'UNESCO ainsi que les conventions et les plans d'action pour les mers régionales.

53. $C f$. les choix du PNUE dans son programme d'action issu d'une décision du Conseil d'administration du PNUE de 1974 (UNEP/GC.26, 22 mars 1974, décision $\mathrm{n}^{\circ}$ 8). Actuellement "Programme pour les océans et les zones côtières ", il réunit 36 conventions et protocoles additionnels relatifs à la protection du milieu marin.

54. M. De.jEANT-Pons : "La Méditerranée en droit international de l'environnement", Economica, Paris, CERIC, Aix en Provence, 1990, (pp. 1-374). Outre la convention de Barcelone, d'autres instruments du même type ont été adoptés dans le cadre du programme pour les océans et les zones côtières du PNUE. 
C'est notamment le cas de la convention de Barcelone pour la protection de la Méditerranée et de son protocole sur les aires spécialement protégées, dont la révision de 1995 a intégré les acquis de la CDB $^{55}$ : les principes de précaution et du pollueur-payeur, l'obligation de réaliser des études d'impact sur l'environnement et le principe d'information et de participation du public. La notion de " biodiversité " apparaît également, sous la forme d'une obligation faite aux États parties de prendre " toutes les mesures appropriées pour protéger et préserver la diversité biologique, les écosystèmes rares et fragiles aussi bien que les espèces de la faune et de la flore sauvages qui sont rares, épuisées, menacées ou en danger, et leurs habitats, dans la zone à laquelle s'applique la Convention " 56 . C'est en vertu de celle-ci qu'a été adopté à Barcelone, le 10 juin 1995, un nouveau protocole relatif aux aires spécialement protégées et à la diversité biologique en Méditerranée, entré en vigueur le 12 décembre 1999.

L'une de ses principales innovations réside dans l'élargissement de son champ d'application à la haute mer, qui marque la reconnaissance d'une protection basée sur des critères scientifiques et écologiques ne s'arrêtant pas aux frontières juridiques ${ }^{57}$. Il s'agit d'abord de protéger des espèces, souvent migratrices, dont les habitats ou les zones de rassemblement et de frai se situent au-delà de la mer territoriale. Or, en application de la partie IX de la CNUDM, cette extension du champ d'application géographique ne pouvait se faire que dans un cadre de coopération régionale. La tâche était d'autant plus difficile qu'aucun accord international ne prévoyait auparavant la possibilité de créer des aires marines protégées en haute mer.

Pourtant, le sanctuaire Pelagos destiné à assurer la protection des mammifères marins en mer de Ligurie, première aire marine protégée de ce type, a vu le jour, à Rome, le 25 novembre 1999 par accord entre la France, l'Italie et Monaco. Émanation directe du protocole de Barcelone, il réussit à articuler les nouvelles obligations introduites par la CDB au cadre juridique posé par le droit de la mer, le sanctuaire étant établi en grande partie dans la haute mer. Son statut "d'aire spécialement protégée d'importance méditerranéenne " permet de rendre opposables à tous les États parties au protocole de Barcelone les mesures adoptées dans le cadre du plan de gestion. Juridiquement, rien n'empêche pourtant les navires des États tiers de ne pas respecter les dispositions adoptées, mais il leur sera relativement difficile, au moins politiquement, de s'opposer aux États riverains.

\subsection{Enjeux pour l'avenir}

Deux problèmes essentiels sont particulièrement difficiles à résoudre, tant ils impliquent des intérêts économiques puissants, les changements climatiques et leur impact sur les récifs coralliens, ainsi que la protection de la biodiversité en haute mer où domine le principe de liberté.

\section{a. Les changements climatiques et le cas particulier des récifs coralliens}

Les changements climatiques affectent de manière négative le milieu marin, alors que le rôle régulateur des océans est maintenant reconnu. Dans ce secteur,

55. "L'esprit de Barcelone et l'esprit de Rio se rencontrent dans cette révision ", M.A. TAVOSO : " La Convention de Barcelone pour la protection de la mer Méditerranée contre la pollution et le développement durable ", MAP Technical Reports Series, n 117, UNEP, Athènes, 1997, p. 50.

56. Article 10 de la convention de Barcelone, portant spécialement sur la conservation de la biodiversité.

57. Article 2, $\$ 1$ du protocole de Barcelone (1995). 
la COP a adopté des recommandations générales ${ }^{58}$, mais elle a concentré son effort sur des écosystèmes spécifiques, en particulier sur le blanchiment des récifs coralliens ${ }^{59}$. Ces récifs qui ne couvrent que $1 \%$ de la surface des mers mais abritent un tiers des poissons marins et probablement un demi-million d'espèces, sont particulièrement menacés aujourd'hui, tant par l'augmentation de la température des mers que par toutes les autres pressions anthropiques. Or, on estime qu'ils jouent un rôle de nourricerie essentiel à la chaîne alimentaire. Ils font donc l'objet d'un plan de travail adopté par la quatrième conférence des parties, coordonné avec les autres actions internationales, - Initiative internationale en faveur des récifs coralliens, UNESCO, programme pour les mers régionales... En 2006, la huitième COP s'est donné pour but d'aider les petits États insulaires, qui sont les premiers concernés avec, généralement, peu de moyens.

Un mouvement s'est dessiné en faveur de larges protections des récifs, à partir de l'expérience inaugurale du classement au patrimoine mondial de l'UNESCO de la grande barrière de corail australienne : quatre cents espèces de coraux, mille cinq cents espèces de poissons et des espèces phares comme la grande tortue verte ${ }^{60}$. La France y participe par l'Initiative française pour les récifs coralliens dans le Pacifique Sud et l'on peut espérer le classement de $60 \%$ de la superficie du lagon de Nouvelle-Calédonie, le principe en ayant été accepté à la fin de 2006 : récifs coralliens, mangroves et herbiers comme écosystèmes associés, plan de gestion global, plans de gestion participative pour chacun des sites; l'examen par l'UNESCO est prévu en 2008. L'efficacité de ces classements dans un contexte global dégradé reste à démontrer.

\section{b. La protection de la biodiversité en haute mer}

L'exploration du fond des océans au cours des trente dernières années a révélé une biodiversité inattendue, tant par sa richesse que par son taux d'endémisme. C'est notamment le cas des monts sous-marins dont les contreforts concentrent les particules organiques et le plancton qui démarrent la chaîne alimentaire : invertébrés, coraux, éponges, concombres de mer, crevettes. Le sommet de ces monts sous-marins est couvert d'algues et d'organismes vivants enrichissant les eaux situées à proximité et procurant une source de nourriture aux tortues marines, cétacés ou aux grands poissons pélagiques tels que les espadons ou les thons. Tout cela est d'autant plus fragile que le cycle de reproduction des espèces qui les peuplent est particulièrement lent. Certaines espèces de poissons d'eaux profondes vivent jusqu'à cent cinquante ans et n'atteignent leur maturité sexuelle qu'au bout d'une trentaine d'années.

La principale menace qui pèse sur ces écosystèmes est le développement actuel des activités de chalutage en haute mer. Les progrès technologiques conduisent à racler les fonds marins au détriment de ces écosystèmes riches et fragiles. Or, en vertu du principe de liberté de navigation en haute mer, il n'est pas possible de réglementer ces activités. Les commissions de pêche offrent toutefois des solutions pour concilier les obligations contradictoires en ouvrant un espace de discussion entre États pour réglementer certains types de pêche à une

58. La décision VIII/30 adoptée à Curitiba (Brésil) en mars 2006, retient cette approche transversale et globalisante.

59. Ce phénomène très inquiétant repose sur la détérioration du lien entre les coraux et les zooxanthelles, qui disparaissent progressivement alors qu'elles absorbaient le $\mathrm{CO}^{2}$ libéré par les coraux et leur fournissaient en retour divers nutriments.

60. Malgré ces efforts consentis depuis 1981, le GIECC a récemment fait savoir qu'il estimait qu'elle aurait disparu d'ici vingt ans. 
échelle régionale. Ainsi, la commission générale des pêches pour la Méditerranée (CGPM) a décidé en février 2005, sur la base de considérations scientifiques, d'interdire les activités de pêche dans les eaux méditerranéennes à plus de 1000 mètres de profondeur ${ }^{61}$. Cette recommandation, la première de ce type dans le monde, a pris effet à partir de juin 2005 .

Au cours des dix dernières années, la communauté internationale a perçu l'urgence d'une action coordonnée en faveur de la protection du milieu marin audelà des seules zones côtières comme le montrent plusieurs textes déclaratifs, ainsi que les COP 7 et 8 de la CDB ${ }^{62}$. Les actions concrètes sont toutefois rares.

\section{B. Des objectifs périphériques qui obtiennent quelques résultats}

Ce n'est pas le cœur de la convention qui a le mieux avancé, mais sa périphérie, ce dont on peut offrir trois exemples : les risques biotechnologiques, la bioprospection, les droits intellectuels des peuples autochtones et communautés locales. Ces trois problèmes sont loin d'être réglés, mais ils ont avancé et une mise en œuvre concrète peut être constatée sur certains terrains. Il est difficile d'avancer une explication unique à cette réussite relative. Dans le premier cas, un point de vue cynique n'est pas à exclure : le pays exportateur d'OGM le plus important, les États-Unis, n'a pas signé le protocole de Carthagène, le Canada et l'Argentine ne l'ont pas ratifié et, à l'époque où le Brésil l'a ratifié, il n'était pas exportateur d'OGM. Donc, les États parties au protocole regroupaient les pays importateurs, actuels ou potentiels, entre lesquels il n'a pas été trop difficile de trouver un accord, même si les États exportateurs, intégrés à la négociation malgré leur position extérieure, ont poussé à de multiples compromis. Il faut souligner que la réflexion sur cette pratique devrait être menée rapidement.

Dans les deux autres cas, il s'agit de questions encore plus marginales au regard de la protection de la biodiversité, mais hautement symboliques. On n'a pas dépassé le stade des lignes directrices facultatives, assez consensuelles pour la bioprospection, juste tolérées pour les droits intellectuels des peuples autochtones. Ici, on est au stade d'une "civilisation des pratiques" par l'exemple. Si les nouvelles pratiques gagnent du terrain, il sera peut-être possible de convaincre les organisations internationales compétentes de prendre en compte ces revendications nouvelles, et de sortir de leur position attentiste actuelle. À tout seigneur, tout honneur, il est logique de commencer par le protocole de Carthagène adopté en 2000, entré en vigueur le 11 septembre 2003, qui est à ce jour le seul protocole attaché à la CDB. Il compte aujourd'hui cent quarante et un États parties.

61. Recommandation CGPM/2005/1 concernant la gestion de certaines pêcheries exploitant des espèces démarsales et des espèces vivant en eau profonde, point $2:$ "les membres de la CGPM sont tenus d'interdire l'utilisation de dragues traînées et le chalutage à des profondeurs supérieures à $1000 \mathrm{~m}$ "; des organismes scientifiques comme le CIESM : commission internationale pour l'exploration scientifique de la mer Méditerranée [www.ciesm.org], ou des ONG comme le WWF, Greenpeace ou l'UICN ont contribué à l'adoption de cette réglementation. Cet exemple pourrait faire école : le gouvernement américain a ainsi désigné en juillet 2006 dans les eaux autours des îles aléoutiennes $\left(957000 \mathrm{~km}^{2}\right)$ une « zone de conservation de l'habitat " au sein de laquelle le chalutage de fond est interdit.

62. Résolution de l'Assemblée générale des Nations Unies n ${ }^{\circ} \mathrm{A} / 57 / \mathrm{L} .48 \mathrm{du} 10$ décembre 2002 à l'occasion du $20^{\circ}$ anniversaire de la signature de la CNUDM sur l'urgence d'une action coordonnée en matière d'aires marines en haute mer ; UICN, $5^{e}$ Congrès mondial des aires protégées, Durban, septembre 2003, recommandation R.5.23: Protecting Marine Biodiversity and Ecosystem Processes through Marine Protected Areas beyond National Jurisdiction. 


\section{Le protocole de Carthagène sur la prévention des risques biotechnologiques}

Le protocole est l'aboutissement d'une revendication qui datait de 1992, puisque dans "Action 21 ", il était demandé de lancer une coopération internationale sur la « biosécurité ". L'article 8 , g, de la convention a repris cette idée et son article 19.3 affirma la nécessité d'un protocole. Il aura pour objet la biosécurité, soit l'évaluation, la prévention et la gestion des risques environnementaux et sanitaires associés aux biotechnologies.

Le développement rapide de ces techniques, dans un nombre limité d'États à grande puissance productive, a dessiné de nouvelles fractures au sein de la société internationale; d'un côté des pays exportateurs, développés mais aussi émergents, de l'autre des pays actuellement ou potentiellement importateurs, PVD et pays développés. L'Europe tient pour le moment une place particulière : potentiellement productrice et exportatrice, elle est encore uniquement importatrice de manière massive à destination de l'alimentation animale et montre une méfiance certaine, qui se traduit par une réglementation très particulière. Il y a donc une alliance naturelle entre les pays exportateurs regroupés dans le groupe dit de Miami, quel que soit leur statut économique ${ }^{63}$. De même, on trouve une alliance entre pays importateurs, quel que soit leur niveau de développement; mais une différence de taille existe entre l'Union européenne et la plupart des pays en développement qui ne disposent pas des moyens d'évaluer ou gérer efficacement ces risques. Le groupe de Miami considère que les restrictions au commerce des OGM ne sont pas justifiées et relèvent du protectionnisme déguisé. Au contraire, l'Union européenne défend une position assez protectrice qui correspond à sa propre législation. À côté, un groupe dit de compromis adopte des positions intermédiaires ${ }^{64}$. L'objectif était tant d'harmoniser des législations nationales disparates, voire inexistantes, que d'organiser la coopération et de prévenir, voire résoudre, d'éventuels conflits interétatiques. Le contenu du protocole, bien que marqué par de multiples compromis et un champ d'application étroit, est assez novateur dans sa conception ${ }^{65}$. Mais les perspectives de mise en œuvre sont fragilisées par la non-participation des États exportateurs, dont la position semble relayée par le droit de l'OMC.

\subsection{Un contenu novateur}

De négociations difficiles est sorti un instrument assez protecteur, conformément aux vœux de l'Union européenne et des pays en développement dont les besoins spécifiques sont pris en compte (e). L'aspect le plus frappant est l'exigence d'un accord préalable pour toute importation, ce qui est en principe contraire à la liberté des échanges (b). D'autre part, l'importance accordée à cet outil crucial qu'est l'étiquetage, également régi par le droit de l'OMC en tant qu'obstacle non tarifaire aux échanges, n'est pas moins révélateur (d). Il reste que le champ d'application est très étroit (a) et que des compromis, imprécisions ou lacunes émaillent l'ensemble du texte.

63. Il regroupe les exportateurs signataires de la CDB : Canada, Australie, Argentine, Chili et Uruguay, soutenus par les États-Unis qui n'ont pourtant qu'un rôle d'observateurs. Quatre ont signé le protocole mais ne l'ont pas ratifié (Argentine, Canada, Chili, Uruguay), l'Australie est signataire de la CDB mais n'a pas signé le protocole.

64. Japon, Mexique, Norvège, Corée du Sud, Suisse, Singapour.

65. M-A. HeRMITTE/C. NoIVIILE : “ Marrakech et Carthagène comme figures opposées du commerce international " in J. BOURRINET/S. MALJEAN-DLBOIS (dir.), La régulation du commerce international des OGM, Paris, La Documentation française, coll. Monde européen et international, 2002, 383 p. 


\section{a. Le champ d'application}

La définition du champ d'application du protocole figurait parmi les enjeux majeurs des négociations, certains souhaitant le limiter à ce qui pouvait menacer la diversité biologique, donc être introduit dans l'environnement, comme les semences. D'autres envisageaient un champ d'application plus vaste, englobant les OGM utilisés pour la nourriture humaine ou animale, directement mais aussi après transformation, ou encore les OGM utilisés pour la médecine. Une solution intermédiaire a été retenue. Le protocole n'emploie pas l'expression usuelle d'organisme génétiquement modifié, mais s'attache aux "organismes vivants modifiés" (OVM) définis comme "tout organisme vivant possédant une combinaison de matériel génétique inédite obtenue par recours à la biotechnologie moderne" (article 3). Est un organisme "vivant" "toute entité biologique cupable de transférer ou de répliquer du matériel génétique, y compris des organismes stériles, des virus et des viroïdes". Le protocole signifie ainsi qu'il ne couvre que les produits biologiquement actifs, comme les semences et les produits agricoles non transformés destinés à l'alimentation humaine ou animale. Les produits dérivés, tels que l'huile, la farine, les sauces tomate, les cufs de poules nourries au maïs transgénique, sont donc exclus du champ d'application du protocole. De même, les OVM pharmaceutiques sont a priori hors champ, même si les parties demeurent libres de les soumettre à une évaluation des risques avant d'accepter leur importation.

b. L'accord préalable en connaissance de cause et le principe de l'évaluation préalable des risques

Cette procédure (APCC) contraint l'État exportateur à notifier par écrit l'exportation d'OVM à l'État importateur, "avant le premier mouvement transfrontière intentionnel " (article 7). La notification doit s'accompagner d'un grand nombre d'informations, dont le contenu minimum est spécifié à l'annexe I. Pour autant, la troisième réunion des parties n'a pas vraiment avancé à cet égard, les parties n'ayant toujours pas trouvé d'accord minimal sur les critères d'identification des OVM destinés à l'alimentation ou à la transformation et cherchant seulement à obtenir un accord sur la nécessité, minimale, de l'utilisation d'une facture commerciale. Celle-ci devrait accompagner les OVM et permettre de les repérer soit par un système de traçabilité de la semence au produit fini (système européen), soit par une analyse finale des cargaisons faite au moment de l'importation, ce qui est moins précis ${ }^{66}$. Actuellement, on s'interroge toujours sur la manière dont les " gouvernements [peuvent garantir] que la documentation [...] stipule clairement le code de l'évènement de transformation de l'OVM ", c'est-àdire le code qui permet de savoir de quel gène il est doté (seul moyen d'en détecter la présence). Cette indécision sur l'identification rend bien difficile le respect du principe selon lequel le pays importateur doit établir ses décisions sur la base d'une évaluation des risques reposant sur des critères environnementaux et de santé publique. Le protocole pose pourtant ici des obligations procédurales précises et fournit par là un outil précieux pour l'harmonisation des procédures d'évaluation scientifique. Élément notable, l'État importateur peut exiger de

66. BS-III/10, sur l'article 18-2 a ; particulièrement le point 4. En fait, deux systèmes s'opposent. L'Union européenne a retenu un système lourd d'évaluation des risques, coexistence des cultures OGM et non OGM et traçabilité généralisée. Les États exportateurs ont au contraire des évaluations préalables plus légères, et surtout ne connaissent pas l'organisation des cultures permettant la coexistence ni l'obligation de traçabilité. Elle peut être organisée sur une base contractuelle à la demande des acheteurs mais demeure alors beaucoup plus aléatoire et coûteuse. 
l'exportateur qu'il réalise à ses frais l'analyse des risques, ce qui constitue un certain renversement par rapport aux procédures traditionnelles du commerce international (article 15).

L'État importateur doit donner son consentement par écrit, et il peut refuser l'importation, la conditionner, demander des renseignements supplémentaires, voire prolonger le délai de réflexion ou suspendre l'importation si un risque apparaît. Cette possibilité a de l'importance dès lors que la procédure APCC ne vaut que pour le premier mouvement d'un OVM.

La procédure APCC, particulièrement lourde et contraignante, s'applique aux OVM agricoles destinés à être introduits directement dans l'environnement (semences, poissons vivants). En revanche, en sont exclus les produits pharmaceutiques, les OVM en transit ou destinés à être utilisés en milieu confiné ou encore les OVM que la Conférence des parties aura définis comme " peu susceptibles d'avoir des effets défavorables sur la conservation et l'utilisation durable de la diversité biologique, compte tenu également des risques pour la santé humaine" (article 7). De manière plus significative encore, les OVM destinés directement à l'alimentation humaine ou animale ou à la transformation se verront appliquer une procédure beaucoup plus souple définie à l'article 11 , conformément à la demande du groupe de Miami et des États-Unis.

\section{c. La reconnaissance du principe de précaution}

Après d'âpres débats avec le groupe de Miami, l'Europe avait obtenu l'inscription du principe dans les articles 9 et 10, lui donnant un contenu opérationnel puisqu'il pouvait être invoqué pour refuser l'importation d'OVM agricoles ou destinés directement à l'alimentation ou la transformation. Se posait dès lors la question de l'articulation de ces dispositions avec le droit de l'OMC. En effet, dans l'affaire des hormones, l'Europe avait demandé sans succès l'application d'un principe de précaution dit " exogène au droit de l'OMC ". Arguant des incertitudes sur sa valeur juridique, notamment au niveau international, l'Organe d'appel avait fait preuve d'une grande prudence, considérant comme " superflu, et probablement imprudent, [de prendre] position dans le présent appel au sujet de cette question importante, mais abstraite " 67 . Le rapport du groupe spécial qui a statué dans l'affaire des produits biotechnologiques va dans le même sens. Même s'il admet que le contexte a évolué depuis 1998 , le groupe spécial considère que "le statut juridique du principe de précaution reste incertain " et juge plus prudent de s'abstenir de prendre position sur ce point ${ }^{68}$. Quoi qu'il en soit, le droit de l'OMC n'ignore pas totalement le principe ou tout au moins construit sa propre approche des mesures que les États sont autorisés à prendre en présence d'incertitudes scientifiques. La jurisprudence a d'ailleurs ouvert de nombreuses brèches, permettant à certains de conclure que "l'autonomie dont les membres de l'OMC bénéficient dans la définition de leurs propres niveaux de protection sanitaire et environnementale rend " même "l'invocation d'un principe de précaution exogène inutile» 69 .

67. Rapport de l'Organe d'appel, Communautés européennes - Mesures concernant la viande et les produits carnés, WT/DS26/AB/R, 12 octobre 1998, $\$ \$ 123-124$.

68. § 7.89.

69. L. GRADONI/H. Ruiz FABRI, "Droit de l'OMC et précaution à la lumière de l'affaire des "OGM" ", in "Principe de précaution", dossier coordonné par C. NoIvili.E, Recueil Dalloz, 2007, 22 ; H. RUIZ FABRI, "L'OMC et le principe de précaution ", in La décision publique et le droit de la responsabilité face au principe de précaution, RJE, $\mathrm{n}^{\circ}$ spécial, 2000, pp. 55-66; C. NOIVILLE, Du bon gouvernement des risques, PUF, Les voies du droit, Paris, 2003, p. 138. 


\section{d. L'étiquetage}

L'épineuse question de l'étiquetage a failli faire échouer les négociations à Montréal. Le groupe de Miami s'est battu jusqu'au bout pour éviter la séparation et l'étiquetage des filières OVM et non OVM, alors que l'Union européenne maintenait la position inverse. En se fondant autant sur la nécessaire liberté de choix des consommateurs que sur des exigences de sécurité sanitaire et environnementale, les pays européens ont fait leur cheval de bataille de l'étiquetage des OGM, non seulement pour les bactéries, animaux ou plantes génétiquement modifiés eux-mêmes, mais aussi pour les produits agricoles ou alimentaires qui en résultent. Le droit communautaire impose déjà des obligations d'étiquetage pour les produits alimentaires, les additifs, les arômes et l'alimentation animale ${ }^{70}$, et doit être prochainement complété concernant les semences qui devraient bénéficier d'un seuil plus bas. La traçabilité, qui permet de sécuriser des contrôles, par nature difficiles et coûteux, repose sur l'organisation de filières séparées dès l'origine, selon la recommandation du 23 juillet $2003^{71}$. Elle ne peut toutefois être mise en œuvre efficacement si l'étiquetage ne constitue pas une obligation internationale. De récentes contaminations accidentelles l'ont mis en lumière, qui ont entraîné une suspension de l'intégralité des importations de riz américain par exemple ${ }^{72}$. L'Union européenne avait donc tout intérêt à ce que le protocole pose des obligations claires en la matière. Finalement, sur ce point aussi, un compromis a été trouvé : les OVM destinés à être utilisés directement pour l'alimentation humaine ou animale ou à être transformés doivent être étiquetés : "peuvent contenir des OVM ". La formule laisse la possibilité de donner plus d'informations, mais le protocole ne contient aucune obligation de préciser la nature des produits, ni de garantir la présence ou l'absence d'OVM. La difficulté a été repoussée et continue de l'être à chaque réunion : le protocole laissait à la réunion des parties deux années après l'entrée en vigueur du protocole pour décider ultérieurement d'exigences plus spécifiques et détaillées pour l'identification des OVM (article 18). Or, la première réunion des parties (REP) a mis en évidence de nombreux blocages que l'on retrouve dans d'autres espaces de négociation, comme la commission du Codex alimentarius chargée de l'élaboration des normes techniques internationales dans le domaine de la sécurité alimentaire. Le protocole est un peu plus précis pour les OVM destinés à une utilisation en milieu confiné, qui doivent être clairement identifiés ; les règles de sécurité à observer pour le transport, le stockage et l'utilisation doivent aussi être précisées. Enfin, des obligations relativement lourdes sont définies pour les OVM destinés à être introduits intentionnellement dans l'environnement, comme les semences.

70. Étiquetage obligatoire au-delà de $0,9 \%$ pour tous les OGM et les produits issus d'OGM comme le sucre et l'huile, tous les produits alimentaires étant soumis à l'obligation de traçabilité. Voy. les règlements (CE) $n^{\circ} 1829 / 2003$ du Parlement européen et du Conseil du 22 septembre 2003 concernant les denrées alimentaires et les aliments pour animaux génétiquement modifiés, JOCE $\mathrm{n}^{\circ} \mathrm{L} 168 / 1 \mathrm{du}$ 18 octobre 2003, et (CE) $n^{\circ} 1830 / 2003$ du Parlement européen et du Conseil du 22 septembre 2003 concernant la traçabilité et l'étiquetage des organismes génétiquement modifiés et la traçabilité des produits destinés à l'alimentation humaine ou animale produits à partir d'organismes génétiquement modifiés, et modifiant la directive 2001/18/CE, JOCE, ${ }^{\circ}$ L 268 du 18 octobre 2003, p. 24.

71. Recommandation de la Commission du 23 juillet 2003 établissant des lignes directrices pour l'élaboration de stratégies nationales et de meilleures pratiques visart à assurer la coexistence des cultures génétiquement modifiées, conventionnelles et biologiques.

72. Par exemple, la décision de la Commission du 5 sept. 2006 relative à des mesures d'urgence concernant la présence de l'OGM non autorisé, LI, rice 601, JOIJE L244/27 du 7 septembre 2006. 


\section{e. La prise en compte des besoins des pays en développement}

Le principe des "responsabilités communes mais différenciées " n'a pas été repris par le protocole, en dépit de la volonté des pays en développement. Mais, inquiets des conséquences économiques et sociales des biotechnologies, ils ont obtenu de pouvoir tenir compte de considérations socio-économiques lorsqu'ils prennent leurs décisions d'importation dans le cadre du protocole (article 26). Beaucoup d'entre eux n'ayant pas les moyens de gérer la biosécurité, ils ont obtenu le principe du renforcement de leurs " capacités ", selon le jargon onusien, outre la possibilité de faire financer les études de risques par l'exportateur.

\subsection{Une mise en ouvre incertaine}

De nombreux points déjà évoqués appellent une concrétisation par la Réunion des parties, principalement tout ce qui a trait aux contrôles et aux sanctions (a). L'incertitude majeure concerne l'articulation entre le protocole et le droit de l'OMC (b).

\section{a. Contrôle de la mise en œuvre et règlement des différends}

Dans son article 29, le protocole est laconique. Lors de sa première réunion, la REP a adopté des procédures de "non-conformité ", nommées Procédures et mécanismes de respect des obligations, organisant le contrôle de la mise en œuvre. Mais elles demeurent très limitées. Le contrôle de type peer-review revêt un caractère très politique, marginalisant le secrétariat et les ONG. Les procédures sont en outre peu précises quant à d'éventuelles sanctions qui iraient audelà de l'effet name and shame.

L'article $34 \mathrm{du}$ protocole, en semblant établir un pont avec le mécanisme de règlement des différends de la convention de Rio, ouvre en complément la porte à des mises en cause de la responsabilité des États, sous une forme plus classique. Celles-ci peuvent d'ailleurs être plus adaptées, dans certaines circonstances, par exemple lorsque les différends sont très «bilatéralisés». Mais le mécanisme de la cnvention de Rio n'est lui-même guère contraignant.

Fnfin, la mise en place d'un régime particulier de responsabilité et de réparation des opérateurs privés est également envisagée, mais sa forme - instrument contraignant ou simples lignes directrices - et plus encore son contenu, restent largement à déterminer ${ }^{73}$. On ne peut pas, là non plus, être optimiste, sachant que l'Union européenne elle-même ne souhaite pas avancer sur ce thème, sauf pour ce qui concerne les dommages à la diversité biologique protégée, qui sont en principes réglés par la directive sur la responsabilité environnementale, mais dont la réalisation concrète reste douteuse, contrairement aux dommages à la santé et aux dommages économiques ${ }^{74}$.

73. $C f$. le rapport du groupe de travail spécial à composition non limitée d'experts juridiques et techniques sur la responsabilité et la réparation dans le cadre du protocole de Cartagena sur la prévention des risques biotechnologiques sur les travaux de sa deuxième réunion, UNEP/CBD/BS/COP-MOP/3/10, 24 février 2006.

74. La directive sur la responsabilité environnementale du 21 avril 2004, JOCE $143 / 56 \mathrm{du} 30$ avril 2004 vise bien les OGM, mais seulement pour les dommages à la diversité biologique protégée, aigles de Bonelli ou autres espèces protégées. Or, si les OGM devaient causer un dommage, ce serait bien plus probablement à la diversité biologique banale, en rendant par exemple, les mauvaises herbes et les insectes résistants aux herbicides et pesticides, ce qui est en cours de réalisation. 


\section{b. Le protocole et le droit de l'OMC}

Situé au carrefour du droit international de l'environnement et du droit du commerce international et prévoyant, comme d'autres accords environnementaux multilatéraux (AEM), des restrictions aux échanges, le protocole conduit inévitablement à s'interroger sur ses relations avec les accords de l'OMC. Le comité du commerce et de l'environnement de l'OMC créé en 1994 à l'issue de la conférence de Marrakech, s'est intéressé de près à la question de leur licéité. Mais contrairement à des AEM plus anciens, le protocole de Carthagène a été adopté six années après l'entrée en vigueur des accords de Marrakech. Le droit de l'OMC et les premières "jurisprudences" rendues dans des affaires intéressant la santé ou l'environnement par les groupes spéciaux, voire l'Organe d'appel, étaient donc en toile de fond des négociations.

Au terme de négociations difficiles, la question n'a pas été tranchée : les pays exportateurs d'OGM n'ont pas fait admettre la subordination du protocole aux règles du commerce international, et les autres n'ont pas réussi l'opération inverse : le protocole refuse d'établir une hiérarchie entre les deux systèmes juridiques, ce qui laisse non résolues les difficultés pratiques que pourrait causer leur coexistence. Au contentieux, l'issue est incertaine, l'incertitude étant aggravée par la supériorité des mécanismes de règlement des différends de l'OMC sur ceux du protocole ${ }^{75}$. La plupart des conflits risquent donc d'être réglés à l'OMC. Or, pour utiles et effectives qu'elles soient, les procédures OMC n'en présentent pas moins l'inconvénient d'appliquer prioritairement le droit de l'OMC 76. L'affaire introduite par les États-Unis, suivis par le Canada et l'Argentine contre l'Union européenne à propos du moratoire que l'Union européenne a observé de 1999 à $2003^{77}$, a donné lieu à quelques solutions, malgré la volonté d'éviter plusieurs questions importantes, mais elles restent incertaines faute d'appel ${ }^{78}$.

La première concerne l'applicabilité du protocole de Carthagène dans l'hypothèse où s'affrontent des États qui sont membres de l'OMC et parties au protocole, et d'autres qui ne sont que membres de l'OMC ou qui ont signé le protocole sans le ratifier. Pour ces derniers, parmi lesquels figurent l'Argentine et le Canada, les Communautés européennes soutenaient que le protocole les lie en vertu de l'article 18 de la convention de Vienne sur le droit des traités qui oblige un État qui a signé un traité à s'abstenir d'actes qui le priveraient de son objet et de son but. S'agissant des États-Unis, les Communautés soutenaient qu'ils participent aux mécanismes d'échange prévus dans le protocole, ce qui amènerait à considérer qu'ils n'ont aucune objection à son endroit. Le protocole, qui serait l'accord international le plus pertinent, repose sur le concept de "soutien mutuel entre les accords sur le commerce et sur l'environnement». Les deux textes devraient donc être interprétés d'une manière " mutuellement compatible ". Or,

75. À la différence de celles du protocole, les procédures de l'OMC peuvent être déclenchées unilatéralement et aboutissent, dans des délais relativement brefs, à une décision obligatoire.

76. Même si l'Organe d'appel a considéré qu'il ne fallait pas lire les accords de l'OMC en les "isolant cliniquement " du droit international public (États-Unis - Normes concernant l'essence ancienne et nouvelle formule, rapport de l'Organe d'appel, WT/DS2/AB/R, 29 avril 1996, p. 19) ou encore, dans l'affaire Crevettes, interprété le GATT à la lumière du droit international de l'environnement ( $\dot{E}$ tats-Unis - Prohibition à l'importation de certaines crevettes et de certains produits à base de crevettes, WT/DS58/ $\mathrm{AB} / \mathrm{R}, \S \S 125$ et ss.). mais.

77. Il faut rappeler qu'il s'agit d'un moratoire sur les cultures, pas sur les importations de soja ou

78. H. GHERARI : "Le conflit sur les produits biotechnologiques tranché à l'OMC ", $R M C, \mathrm{n}^{\circ} 503$, 2006 , p. 654 . 
le groupe spécial (GS) a considéré que ni la convention ni le protocole ne sont des " règles pertinentes du droit international applicables dans les relations entre les Parties " ( $\$ 7.75)$. Cette interprétation ne tient pas compte du fait que le protocole a été adopté par cent trente États par consensus et est rapidement entré en vigueur. Les appels répétés du comité du commerce et de l'environnement ${ }^{79}$, mais aussi de l'Organe d'appel en faveur de la coopération multilatérale dans le domaine de l'environnement, pouvaient laisser espérer un résultat plus constructif ${ }^{80}$. Néanmoins, d'autres éléments de la décision sont plus encourageants. Le premier réside dans une interprétation qui n'avait rien d'évident: pour pouvoir affirmer que la question des contaminations peut donner lieu à des mesures sanitaires qu'un État peut prendre sous condition de validité scientifique pour protéger son territoire, le GS a jugé que les flux de gènes passant d'une plante transgénique, même dûment autorisée, vers une plante non transgénique, pouvaient être assimilés à des "parasites" et donc justifier une mesure (points 7240 et sv.). Il suffit que le flux concerne un exploitant qui ne le souhaite pas (point 7244) ou qu'il risque de provoquer le développement de résistances à un herbicide ou produise des effets sur les " organismes non cibles " ou provoque des pertes économiques pour les agriculteurs conventionnels (points 72583, 72636, $72630,72640)^{81}$.

De plus, le GS a eu une approche souple de la question des moratoires. S'ils ont été jugés trop longs, ils ne sont pas condamnés en tant que tels. Ils peuvent donc être un outil de gestion des risques à la condition d'avoir une motivation scientifique sérieuse et d'être mis à profit pour en sortir le plus rapidement possible. Sans que l'on puisse être très assuré du résultat, on peut affirmer que le protocole a été purement et simplement écarté, mais que son contenu n'a pas été totalement oublié dans l'interprétation donnée à l'accord SPS : le principe de coexistence entre les filières OGM et non OGM n'a pas été récusé en tant que tel, ce qui explique que les " contaminations " aient été jugées comme un évènement indésirable au même titre qu'une maladie des plantes.

Comme le reste de la CDB, le protocole représente un effort juridique important qui peine à trouver ses applications concrètes. C'est un peu l'inverse pour les contrats de bioprospection; des modèles contractuels diffusent progressivement, qui tentent de réaliser un certain nombre d'objectifs d'intérêt général. Il n'y a donc pas de protocole, mais une relative réussite pratique.

\section{Les contrats de bioprospection}

Lorsque la $\mathrm{CDB}$ fut négociée, les médias avaient commencé à dénoncer une distorsion des échanges. D'un côté, le développement des biotechnologies mettait en lumière l'intérêt économique des ressources génétiques; la biodiversité était alors plus clairement perçue comme une réserve de molécules aux propriétés pharmacologiques ou insecticides qui, par le biais conjugué de la technique et du droit des brevets ${ }^{82}$, étaient devenues valorisables. De l'autre, en vertu d'une

79. Rapport (1996) du comité du commerce et de l'environnement, WT/CTE/1, 12 novembre 1996 , $\$ 171$, section VII du rapport du conseil général à la conférence ministérielle de 1996, WT/MIN(96)2, 26 novembre 1996.

80. Voy. par exemple la deuxième affaire des "crevettes", Rapport de l'Organe d'appel du 22 octobre 2001 , WT/DS58/AB/RW.

81. Cf. le travail de mémoire de Jose Corti Varela [http://panjuris. univ-paris1.fr/].

82. CF. la directive $98 / 44 / \mathrm{CE}$ du 6 juillet 1998 relative à la protection juridique des inventions biotechnologiques; pour le droit français, la loi n ${ }^{\circ} 2004-800$ du 6 août 2004 relative à la bioéthique (art. 17) et la loi n 2004-1338 du 8 décembre 2004 relative à la protection des inventions biotechnologiques. 
pratique traditionnelle, universités et entreprises des pays développés prospectaient librement ces ressources de par le monde et les plaçaient en collections, à des fins purement scientifiques et/ou commerciales, la frontière entre les deux étant poreuse; le droit positif n'accordait aucune valeur économique à la ressource à son point d'origine, tandis que le droit des brevets permettait de valoriser le produit qui en était tiré.

C'est pour lutter contre un système ainsi perçu comme inéquitable que la CDB renonça au concept de patrimoine commun de l'humanité, corrélé à un libre accès gratuit de tous à la ressource. Il fallait que les États du Sud, généralement riches en biodiversité, pussent en maîtriser l'accès et prétendre à une part des avantages escomptés par les États riches en technologies, le plus souvent localisés au nord. Pour ce faire, l'article 15.1 de la CDB avait posé le principe de la souveraineté des États sur leurs ressources naturelles pour en déduire "le pouvoir de déterminer l'accès aux ressources génétiques "; celui-ci appartiendrait désormais aux gouvernements et serait régi par la législation nationale ; l'article 15.5 ajoutait que "l'accès aux ressources génétiques est soumis au consentement préalable donné en connaissance de cause de la Partie contractante qui fournit lesdites ressources, sauf décision contraire de cette Partie ".

Le système conçu en 1992 impliquait donc que les législateurs du Sud déterminassent les règles d'accès aux ressources en ménageant, via le consentement préalable, une place centrale à l'outil contractuel appelé à organiser les modalités précises de cet accès qui peut évidemment varier selon les circonstances. C'est ainsi que l'article 15.4 de la convention stipulait : "L'accès, lorsqu'il est accordé, est régi par des conditions convenues d'un commun accord [...]", lesquelles doivent, en vertu de l'article 15.7, assurer "le partage juste et équitable des résultats de la recherche [...] et des avantages résultant de l'utilisation commerciale et autre des ressources génétiques avec la Partie contractante qui [les] fournit ". Partage des avantages et équité internationale, au cœur de la CDB, reposaient donc sur la loi autant que sur le contrat.

Or si des lois ont bien été votées en nombre non négligeable, certaines sont trop sommaires ; tous les États ne s'en sont par ailleurs pas dotés. C'est alors le contrat qui occupe une place centrale dans l'échange de biodiversité ${ }^{83}$. Tel qu'appréhendé par les rédacteurs de la convention, il poursuit deux objectifs d'intérêl général. Le premier est d'inciter les États fournisseurs à conserver leur biodiversité, ce qu'illustre bien l'arrêt Yellowstone rendu le 12 avril 2000 par la District Court du District de Columbia ${ }^{84}$. À l'origine du contentieux, un contrat conclu entre une entreprise de biotechnologie, Diversa, et le parc national américain Yellowstone : en contrepartie du droit qu'elle acquérait de collecter et d'évaluer la diversité microbienne du parc, Diversa s'engageait à acquitter un

83. Voy. notamment K. TEN KATE/S. LAIRD, The Commercial Use of Biodiversity: Access to Genetic Resources and Benefit-Sharing, Earthscan Publications, Londres, 1999 ; S. LAIRD (dir.), Biodiversity and Traditional Knowledge: Equitable Partnerships in Practice, Earthscan Publications, Londres, 2002 ; J.-F. MORIN, "Les accords de bioprospection répondent-ils aux objectifs de la Convention sur la diversité biologique? ", Revue de droit de l'Université de Sherbrooke, vol. 34, n 1 , novembre 2003 , pp. 307 et s. ; F. BEI.LIVIER, "Les contrats portant sur les ressources génétiques végétales : typologie et efficacité ", in M.-A. HERMITTE (dir.), Les ressources génétiques végétales et le droit dans les rapports Nord-Sud, Bruylant, 2004, pp. 193 et s.

84. Même si la bioprospection était ici de nature particulière puisque la biodiversité du parc Yellowstone étant endémique, elle conférait notamment à la direction de ce dernier un pouvoir de négociation sans commune mesure avec celui de "fournisseurs classiques " de diversité biologique, sauf lorsqu'ils appartiennent à des régions à fort endémisme comme Madagascar. On remarquera que les États-Unis, qui n'ont pas ratifié la CDB, mettent en ceuvre son esprit, défendant la position selon laquelle le contrat suffit à satisfaire ses objectifs. 
"droit d'entrée " de 20000 dollars par an, à fournir diverses techniques et matériels à son co-contractant et à lui verser 5 à $10 \%$ de redevances sur toute commercialisation ultérieure de produits. Saisi de la légalité d'un tel accord, le juge estima que non seulement il n'était pas contraire à la réglementation des parcs nationaux mais que, loin de relever d'une politique critiquable d'industrialisation des parcs, le contrat constituait un outil d'appoint utile à la mise en œuvre de la réglementation, un élément moteur du financement des parcs et, plus globalement, de conservation de la nature. Que le contrat de bioprospection joue ce rôle entraîneur pour l'ensemble de la biodiversité, au-delà de ces sanctuaires que sont les parcs nationaux, tel était bien l'objectif poursuivi par les rédacteurs de la CDB.

Le contrat de bioprospection devait ensuite éviter le pillage des ressources biologiques et placer les échanges de biodiversité sous le sceau de l'équité. Chercheurs et industriels n'auraient plus la liberté de collecter, breveter et exploiter plantes, écorces ou micro-organismes sans contrepartie pour les populations autochtones, eussent-elles joué un rôle notable dans le développement de l'invention en fournissant par exemple des indications sur les propriétés cicatrisantes d'une herbe utilisée dans la pharmacopée locale depuis des siècles ${ }^{85}$. Même si la biodiversité n'est pas l'or vert que l'on fait parfois miroiter, un partage des fruits de la prospection s'imposait, non seulement au regard de l'impératif de justice mais aussi eu égard à la nécessité plus prosaïque de maintenir ouvert aux chercheurs et à l'industrie le vivier de la biodiversité.

Quinze ans plus tard, le contrat de bioprospection est couramment utilisé, son développement confirmant son particularisme $(2.1)^{86}$; mais il ne paraît pas tenir toutes les promesses placées en lui, la nécessité de l'encadrer s'imposant alors (2.2).

\subsection{Le particularisme du contrat de bioprospection}

Le contrat de bioprospection est atypique à deux égards. D'abord, s'il remplit une fonction très classique pour un contrat, à savoir l'échange économique, c'est de manière originale qu'il l'assume, tant en raison de la multiplicité des intervenants et des objectifs poursuivis que du particularisme de son objet. Il s'agit en effet non pas tant de céder des ressources biologiques que de permettre au cédant de s'assurer un contrôle sur les bénéfices qui en seront tirés, dans un avenir probablement lointain (a) ; ensuite, une autre fonction, pour le coup plus inédite, lui est assignée, puisqu'il contribue à une forme de justice distributive (b).

85. Si le thème de la “ biopiraterie " s'est exagérément amplifié, quelques exemples emblématiques témoignent de sa réalité : on pense, entre autres, à l'affaire du " haricot jaune ", développé de longue date par les mexicains puis collecté par un sélectionneur américain qui, après l'avoir fait breveter aux ÉtatsUnis, a subordonné au paiement d'une taxe toute exportation vers ce pays, les agriculteurs du Mexique ne pouvant alors plus librement exploiter ce qu'ils avaient pourtant été les premiers à fabriquer. V. US Patent No. 5,894,079, "Field bean cultivar named enola", actuellement soumis au Board of Patent Appeals and Interferences. Voy. aussi les brevets sur des utilisations fongicides et insecticides du margousier, arbre cultivé en Asie pour ses vertus médicinales et agricoles, dont l'un (brevet européen n 0436 257) a été révoqué le 10 mai 2000 par l'Office européen des brevets pour défaut de nouveauté.

86. Pour des études de cas, voy. sur le site de la CDB / www.biodiv.org/programmes/socioeco/benefit/ case-studies. aspl et, sur le site de l'Organisation mondiale de la propriété intellectuelle, [www.wipo.int/ $\mathrm{tk} / \mathrm{fr} /$ databases/contracts/index.htmll. 


\section{a. Un instrument adapté au particularisme de l'échange des ressources biologiques}

Organiser la collecte, la recherche et les modalités d'exploitation des éventuels résultats, tel est l'objet juridique du contrat de bioprospection. Le premier à avoir fait parler de lui était celui qu'avaient conclu l'entreprise pharmaceutique américaine Merck et l'Institut de recherche scientifique non gouvernemental et non lucratif costaricain Inbio, afin de mettre à la disposition de l'entreprise des plantes et micro-organismes rares constituant autant de matières premières pour de nouveaux médicaments potentiels. Depuis, plusieurs générations de contrats ont contribué à sophistiquer le modèle : le fournisseur et l'entreprise, seuls contractants à l'origine, ont été rejoints par de nombreux intermédiaires participant aux opérations de collecte et dont les statuts sont très divers - jardins botaniques prospectant pour le compte du Nutional Cancer Instilute américain, universités, instituts de recherche locaux, communautés locales, entreprises ou ONG. De cette multiplicité de parties découle nécessairement une structure contractuelle complexe.

Sont alors nés, au gré des besoins, des ensembles de contrats bilatéraux (un pays fournisseur contracte avec une université ou un institut de recherche, qui se lie lui-même à un organisme de recherche ou à une entreprise), des contrats "en roue" (un acteur est lié avec une multitude de co-contractants n'ayant de leur côté aucun lien contractuel entre eux), des contrats emboîtés, des " consortia ".

La caractéristique majeure du contrat de bioprospection réside malgré tout dans son objet. Soit d'abord l'objet juridique : il ne s'agit pas de vendre ni d'échanger des ressources en tant qu'objets matériels, car le fournisseur perdrait alors tout droit sur les résultats qui pourraient en être tirés ultérieurement. Or c'est bien l'objet du contrat de bioprospection que de "définir les conditions d'approvisionnement [...] en matériel, les conditions de collaboration entre [les parties] ainsi que les modalités d'exploitation [...] des résultats [...] issus des recherches que [le prospecteur] entend mener sur lesdits matériels" ${ }^{87}$. Soit ensuite l'objet matériel du contrat - la ressource biologique : celle-ci peut être volée, dupliquée, donner lieu à des recherches cachées, les futurs bénéfices pouvant alors échapper au fournisseur ; sa valeur est en outre aléatoire, le prospecteur n'étant jamais certain d'en tirer un quelconque bénéfice commercial. Dès lors, ces spécificités obligent les parties à rivaliser d'inventivité et impriment au contrat de bioprospection une économie bien particulière qui s'observe à l'étude de ses principales clauses.

Outre les traditionnelles dispositions relatives aux conditions de la prospection et de la recherche (lieu, durée et objet de la collecte, respect des règles de protection de l'environnement, octroi ou non d'une exclusivité temporaire de collecte au prospecteur, confidentialité, etc.), à la loi applicable ou au règlement des différends, quatre principales catégories de clauses se retrouvent régulièrement dans les contrats de bioprospection.

Un premier ensemble concerne les avantages concédés au(x) contractant(s) du pays fournisseur. Colonne vertébrale du contrat, ils sont d'une extrême variété : financiers ou non, immédiats ou à long terme, l'enjeu étant, dans ce dernier cas, de déterminer à quelle part des bénéfices pourront prétendre le ou les fournisseurs des échantillons une fois ces derniers exploités. Les clauses relatives à la propriété industrielle forment un second groupe, indissociable du

87. Pro Natura International/Institut de recherche Pierre Fabre, Accord sur la valorisation de la biodiversité des forêts tropicales du Gabon, 2000. 
premier, et visent à aménager les règles de titularité et d'exploitation des éventuels futurs brevets (les parties en seront-elles co-titulaires? L'un des partenaires les détiendra-t-il seul tandis que l'autre bénéficiera d'une part des redevances?). Ces deux groupes de clauses n'ont de sens qu'au regard d'un troisième, qui organise un contrôle soigneux des recherches et des résultats. Autant l'on conçoit mal que le concessionnaire d'un puits de pétrole vole la ressource à une échelle industrielle, autant rien n'est plus simple et moins visible que de voler quelques micro-organismes ou plantes pour les reproduire en laboratoire ou de prétendre n'avoir rien tiré d'intéressant de la collecte opérée. De nombreux mécanismes sont alors institués pour permettre au fournisseur de suivre à la trace les ressources et leurs utilisations : obligation pour le chercheur de mener son travail sur place ; interdiction faite au prospecteur de céder les échantillons mis à disposition à un tiers, sauf à obtenir préalablement l'accord écrit du cocontractant et à assujettir le destinataire aux mêmes obligations, etc. Enfín, une dernière catégorie de clauses concerne les bénéficiaires du contrat qui, outre les parties, peuvent être l'État fournisseur, certaines populations autochtones lorsqu'elles ont été associées à la prospection comme tiers au contrat, ou un fonds fiduciaire, institution mise en place pour être alimentée par les bénéfices futurs et concilier intérêts privés et intérêt public. C'est alors que le contrat de bioprospection vise à satisfaire des impératifs de justice distributive.

\section{b. Le contrat de bioprospection, vecteur de justice distributive?}

Le contrat de bioprospection est traversé de préoccupations liées à la justice sociale, à la protection de l'environnement - et plus particulièrement de la biodiversité -, à l'aide aux pays en développement, à l'équité des échanges : autant d'impératifs qui lui sont traditionnellement étrangers, lui qui, comme tout contrat, assume théoriquement une fonction commutative, c'est-à-dire une simple fonction d'échange plutôt qu'un rôle de redistribution ${ }^{88}$.

À minima, cette réalité est rendue possible par le fait que le contrat permet la formalisation et la canalisation de pratiques antérieures - quelques contrats ont ainsi pu freiner les prélèvements sauvages. Au-delà, certaines clauses tendent à opérer la conciliation attendue entre conservation et exploitation : clauses imposant des méthodes de collecte respectueuses de l'environnement, affectation d'une part des bénéfices immédiats et futurs à la protection de l'environnement ou dispositions plus originales telles celle qui, dans un contrat conclu au Panama, prévoit que les bénéfices soient également partagés entre un Environmental Trust Fund sur place, le gouvernement panaméen qui s'engage à utiliser ces revenus pour entretenir des parcs nationaux, le prospecteur et les instituts de recherche.

Une observation similaire peut être faite, dans une certaine mesure, en ce qui concerne l'équité des échanges. Certes, des exemples de biopiraterie sont régulièrement mis en lumière, mais l'observation des contrats illustre une civilisation manifeste des pratiques. Universités et centres de recherche qui avaient pris, de facto, l'habitude de travailler avec les universités locales, contractualisent désormais leurs liens avec ces dernières et établissent les conditions de confidentialité, de partage des résultats et de versement d'éventuelles redevances de brevets. Le

88. Sur ce point, of. F. BellivikR/C. NoIVIzLE: Contrats et vivant, LGDJ, 2006, pp. 286 et s. Lintégration des impératifs de justice distributive en droit des contrats, là où on ne l'attend pas forcément, fait aujourd'hui l'objet de vifs débats. On parle aussi dans ce contexte de " justice sociale ". Voir Groupe d'étude sur la justice sociale en droit privé européen, “ Manifeste pour une justice sociale en droit européen des contrats ", RTDciv. 2005, pp. 713 et s. 
rôle de certaines ONG agissant comme intermédiaires, ou de certaines institutions publiques et bailleurs de fonds, s'avère souvent fondamental dans la construction d'un équilibre contractuel ${ }^{89}$. Plus précisément, le versement d'éventuelles redevances à terme est entré dans les mœurs ${ }^{90}$, pendant que les aides immédiates se multiplient : construction d'une école ou d'un dispensaire, association de scientifiques locaux aux opérations de collecte ou transfert des technologies et construction d'équipements permettant à ces derniers de participer aux recherches auxquelles leur biodiversité donne prise ${ }^{91}$. C'est ainsi qu'en vertu d'un contrat d'exploitation de la plante Prunus Africana, l'entreprise Plantecam doit reverser une part de ce qu'elle touche à un fonds de développement des villages et finance un équipement en eau et des formations aux techniques de gestion des ressources végétales.

Pourtant, de nombreuses faiblesses restent perceptibles, qui appellent un encadrement.

\subsection{La nécessité d'un encadrement}

Même s'il est bien tôt pour tenter un bilan des contrats de bioprospection, force est de constater que leur qualité est très inégale, nombre d'entre eux présentant des lacunes, particulièrement au regard de l'objectif d'équité internationale. Une sorte du tutorat, que les lois nationales sur l'accès aux ressources biologiques, trop rares et encore souvent imprécises, peinent à assumer, est recherché dans le cadre de la communauté internationale (a). Toute la difficulté consiste à éviter qu'un tel encadrement, indispensable, n'aboutisse à brider à outrance le contrat et à lui faire perdre, notamment, ses traditionnelles qualités d'adaptation au particularisme des situations (b).

\section{a. Un indispensable tutorat}

Le défaut principal des contrats actuels tient à la conception du partage des avantages, souvent trop sommaire pour protéger réellement les intérêts des fournisseurs ${ }^{92}$. Dans certains cas, la bioprospection échappe purement et simplement au principe de partage. Tel est le cas lorsque le contrat est conclu pour la recherche fondamentale uniquement et que, sans exclure la perspective d'un développement commercial, il s'abstient d'en préciser les conditions. Ainsi, ne sont pas assujettis au partage certains instituts de recherche publics, considérés comme investis de missions d'ordre strictement cognitif justifiant que leur soit transférée gratuitement la ressource. Une telle vision des choses ne correspond pourtant plus à la réalité

89. Voy. le rôle de ProNatura International, ONG qui aide à la mise en place de contrats de partenariat pour l'exploitation équitable de la biodiversité dans certains pays africains ou au Pérou. Voy. aussi le Fonds français pour l'environnement mondial, qui s'est fixé comme règle de ne financer que les bioprospections mettant en œuvre un partage favorisant le développement économique et social local.

90. Voy. le contrat brésilien "Quest. International/F. Alves Correa Neto " dont l'objet est la collecte de plantes à parfum, qui prévoit que toute application industrielle donne lieu au versement de $5 \%$ des bénéfices nets à F. Alves Correa Neto. Voy. aussi l'exemple de la tribu Kani du Kerala (Inde du sud) qui cultive depuis longtemps une herbe aux propriétés anti-fatigue. La tribu reçoit 50 \% des bénéfices perçus par l'Institut public qui a mis au point la technologie et l'a ensuite licenciée à une entreprise pharmaceutique. Voy. U. SCHÜKLENK/A. KIEINSMIDT, "North-South benefit sharing arrangements in bioprospecting and genetic research: a critical ethical and legal analysis ", Developing world bioethics, volume 6, number 3, december 2006, pp. 123 et s., spéc., pp. 128-129: dans ce cas précis, un trust alimenté par les revenus tirés de l'exploitation de la plante en question a été mis en place pour venir en aide de diverses façons à la communauté (assurance pour les femmes enceintes par exemple).

91. Voy. à cet égard, la politique de l'entreprise américaine Diversa décrite par E. Mathur et al. : "An overview of bioprospecting and the Diversa Model ", IP Strategy Today, n 11-2004.

92. Outre le fait que certains accords de bioprospection manquent de force obligatoire lorsqu'ils affectent la forme de lettres d'intention ou autres Memorandum of understanding (MOU). 
puisqu'en pratique, une activité d'inventaire est toujours susceptible de déboucher sur des recherches appliquées en collaboration avec des entreprises privées.

Dans d'autres cas de figure, le partage, bien qu'énoncé en forme de principe, apparaît sujet à caution dans la réalité, et ce pour trois raisons :

- d'une part, sa réalisation est souvent repoussée à l'étape lointaine et aléatoire du développement d'un produit fini. Trop peu de contrats subordonnent la collecte même du matériel biologique à une première contrepartie, alors que ce sera bien souvent la seule compensation effectivement versée dans les cas où la recherche ne débouche sur aucun résultat valorisable ;

- d'autre part, en l'état des pratiques contractuelles, l'impératif d'équité se heurte à la place marginale réservée aux populations locales. Quand bien même elles auront fourni diverses indications - ethnobotaniques par exemple -, elles ne sont généralement pas parties à l'accord, le prospecteur faisant parfois valoir qu'il n'est pas autorisé à contracter avec des entités dépourvues d'existence légale. Au mieux, les communautés locales sont alors simples bénéficiaires tiers au contrat, selon des modalités qui n'établissent pas un rapport véritablement équitable : au-delà de dispositions mineures - remerciements dans les publications scientifiques -, c'est le contractant principal qui capte les avantages à son profit, l'obligation de reverser une part des bénéfices aux populations locales n'étant pas systématique, loin s'en faut. Lorsque les communautés locales figurent bel et bien au contrat en tant que parties, c'est généralement selon des modalités fragiles. Par exemple, elles ne seront engagées qu'avec un seul des différents partenaires (le prospecteur notamment), ces derniers étant de leur côté parties à une structure " en roue " ou "en ligne "; on peut alors s'interroger sur les effets des contrats principaux sur les populations locales étant donné le rapport de force peu favorable à ces dernières ;

- enfin, l'efficacité du partage supposerait des modalités de contrôle qui, en l'état, sont trop rudimentaires pour ne pas fragiliser la mise en ouvre du contrat dans ce domaine où par nature, les difficultés de contrôle sont exacerbées. Comment, en effet, contrôler que tel médicament in fine développé, l'a été dans le respect du principe de partage des avantages avec le fournisseur? En prévision de ces difficultés, certains contrats recourent à diverses clauses classiques (recherches sur place associant des scientifiques locaux, audits, etc.) ou plus subtiles (fourniture de petites quantités d'échantillons dont l'origine et le genre ne sont pas précisément identifiés, de telle sorte que l'industriel ne puisse reproduire la ressource par synthèse ou se la procurer par un autre biais). En dépit de cette inventivité, le contrôle des utilisations reste le plus souvent entravé par un rapport contractuel inégal. Le partage des futurs avantages s'en trouve alors fragilisé d'autant.

En somme, à lui seul, le contrat ne paraît guère à la hauteur de l'impératif d'équité, la réalisation de cet objectif dépendant de l'habileté à négocier du fournisseur, de la bonne volonté de l'acquéreur, d'un investissement intellectuel et d'une compétence encore rares. En l'état, c'est vers la communauté internationale que l'on se tourne pour mettre en place les tuteurs nécessaires pour assainir l'économie de la biodiversité visant à encadrer voire à dépasser le contrat ${ }^{93}$.

93. On ne fera que mentionner ici les travaux de la FAO; ils ne concernent pas le contrat de bioprospection in situ mais l'accord de transfert de matériel par lequel un opérateur sollicite du matériel biologique auprès d'un réseau de banques ex situ. En application du traité international sur les ressources phytogénétiques pour l'alimentation et l'agriculture du 3 novembre 2001 , un “ accord type de transfert de matériel "a été élaboré pour tout accès aux collections de ressources agricoles vitales visées par le traité. Voy. l'accord consultable sur / www. fao. org/ag/cgrfa/French/itpgr. htm] et, sur cette question, C. NoIVILI.E, "Le statut juridique des collections de ressources génétiques ", in M.-A. HERMITTE (dir.), Les Ressources génétiques végétales et le Droit dans les rapports nord-sud, Bruylant, Bruxelles, 2004, pp. 219 et s. 
En premier lieu, elle s'emploie à fixer plus précisément les modalités et le contenu du contrat de bioprospection. Si des lignes directrices ont été adoptées à Bonn à cet effet précis en $2002^{94}$, leur caractère non obligatoire explique l'accent aujourd'hui mis sur la nécessité d'un " régime international sur l'accès et le partage des avantages " qui contraindrait les utilisateurs de matériel biologique à respecter quelques clauses types protectrices des intérêts de l'État et des populations fournisseurs, que ces derniers soient ou non dotés d'une réglementation à cet égard ${ }^{95}$. Plutôt qu'un ordre juridique interne inachevé ou de structure fragile, c'est le droit international qui obligerait ainsi les opérateurs à conclure un contrat, fixerait l'ossature de ce dernier et dicterait les sanctions de son inexécution. Il s'appliquerait aux relations contractuelles, sur la base de la ratification par les États. En second lieu, des négociations portent sur la mise en place et la traçabilité d'un " certificat de conformité " qui devrait être produit par les opérateurs à chaque étape de l'échange et de l'utilisation des ressources, attestant le respect des dispositions législatives ou contractuelles. D'ores et déjà, plusieurs États ont prévu que toute demande de brevet mettant en jeu des échantillons biologiques contienne une mention de leur origine géographique ${ }^{96}$. Enfin, une réflexion a été entreprise dans le cadre de l'Organisation mondiale de la propriété intellectuelle (OMPI) dans le but de forger ex nihilo un droit de propriété intellectuelle sui generis protégeant les savoirs des populations locales sur la biodiversité indépendamment de tout contrat ${ }^{97}$.

Si nécessaire que soit ce triple tutorat, la prudence est de mise car il ne faudrait pas brider les capacités d'adaptation du contrat par un cadre trop contraignant et, pire encore, imposer un carcan tel qu'il justifierait des comportements d'évitement, au besoin par des pratiques illégales.

\section{b. Un tuteur à juste distance}

Si une « loi uniforme " devait guider la matière, encore faudrait-il veiller à ce qu'elle accorde une place à une négociation au cas par cas, seule à même d'exprimer les spécificités économiques propres à chaque secteur - pharmacie, agriculture, industrie cosmétique - ainsi qu'à la situation particulière du lieu de prospection. L'avenir du contrat de bioprospection dépend en effet de la flexibilité qui lui sera ménagée et de sa capacité à ne pas devenir un contrat d'adhésion d'inspiration purement législative. Dans cette veine, même s'ils ne peuvent ni ne doivent se substituer à l'ensemble législatif en cours d'élaboration, les codes de bonne conduite peuvent être utilisés pendant la période de construction de ce dernier ${ }^{98}$. Ils restent en effet un instrument de civilisation des bioprospections et

94. Lignes directrices de Bonn sur l'accès aux ressources génétiques, Décision VI/24, UNEP/CBD/ COP/6/20, avril 2002 [http://www.ige.ch/F/jurinfo/documents/COP-6_F. pdf].

95. Voy. la décision VIII/4, UNEP/CBD/COP/8, mars 2006.

96. Voy. la loi belge du 28 avril 2005 modifiant la loi du 28 mars 1984 sur les brevets d'invention, en ce qui concerne la brevetabilité des inventions biotechnologiques, Moniteur Belge, 13 mai 2005, ou la loi norvégienne $\mathrm{n}^{\circ} 9 \mathrm{du} 15$ décembre 1967 modifiée les 19 décembre 2003, puis 20 mai 2004, consultable sur http://www.patentstyret.no/templates/Page. 699.aspx.

97. Voy. les travaux du comité intergouvernemental de la propriété intellectuelle relative aux ressources génétiques, aux savoirs traditionnels et au folklore, sur le site de l'OMPI [http://www.wipo.int], rubrique "Activités" puis "Savoirs traditionnels - ressources génétiques".

98. Dans ce sens et pour une liste des codes d'ores et déjà existants en la matière, voy. F. BELLIVIER C. NoIVILle, Codes de conduite et équité des échanges de ressources biologiques, IDDRI, Idées pour le débat, $\mathrm{n}^{\circ}$ 10/2006. On ajoutera à cette liste les Guidelines Members on Access to Genetic Resources and Equitable Sharing of Benefits Arising out of their Utilization et le Statement on Access \& Benefit Sharing (ABS) and Disclosure of Origin of Genetic Resources, adoptés le 26 janvier 2007 par la Fédération internationale de l'industrie du médicament. 
constituent un appoint d'autant plus utile aux politiques contractuelles qu'ils sont établis par des acteurs directement confrontés aux particularités de leur champ d'activité.

Ensuite, il faudra être attentif à ce que la complexité d'un contrat trop bien conçu ne soit pas contre-productive. Certains scientifiques se plaignent ainsi de la lourdeur et des coûts entraînés par la négociation du contrat de bioprospection, qu'ils n'ont souvent pas les moyens d'assumer. Ils suggèrent donc, à raison, qu'un contrat type puisse s'appliquer aux premiers stades de la prospection, un contrat plus sophistiqué ne devant être conclu que lors du passage à la recherche commerciale. Il ne faut pas se cacher qu'une part non négligeable des acteurs paraît tentée d'échapper au modèle de la bioprospection tel qu'échafaudé par les rédacteurs de la $\mathrm{CDB}$ et privilégie la figure de la vente. Plutôt que de prospecter elles-mêmes, les entreprises achètent à des "intermédiaires" des échantillons qui paraissent être sans passé, dégagés de tout lien avec un quelconque fournisseur d'origine, qu'il s'agisse d'autorités publiques ou de communautés locales. Dès lors, la recherche qui sera effectuée sur ces échantillons échappera aux tracasseries administratives, au principe de partage des avantages autant qu'à la philosophie même du contrat de bioprospection. Quelles que soient les critiques qu'elles peuvent susciter, de telles pratiques conduisent à se poser sérieusement la question de l'adéquation du modèle actuellement pensé par les législateurs à la réalité et aux contraintes économiques des échanges de biodiversité.

Au bout du compte, le contrat civilise assez bien l'activité de prospection, même si de multiples opérations échappent encore à l'esprit de la convention. Le maillon faible du système concerne manifestement les droits qu'il conviendrait d'octroyer aux populations locales. De ce point de vue, le contrat paraît insuffisant. S'il achoppe souvent en raison de la mauvaise volonté des prospecteurs, il est aussi bien des difficultés liées à la représentation des communautés locales ${ }^{99}$. La solution n'est, alors, sans doute pas de nature contractuelle et l'on peut même douter que le renvoi à la législation nationale soit satisfaisant. Seule, nous semble-t-il, la reconnaissance d'un véritable droit intellectuel reconnu par la communauté internationale permettrait de résoudre le problème.

\section{Les droits intellectuels des peuples autochtones ${ }^{100}$ et des communautés locales}

On vient de le voir, un contrat de prospection a la capacité de protéger les intérêts de toutes les parties en présence quand il est bien conçu. Mais si, en 2007, il n'est plus exceptionnel que l'État fournisseur sache protéger ses intérêts et ceux de ses universités, il délaisse généralement les intérêts des populations qui vivent sur les territoires prospectés. Pourtant, il est fréquent qu'elles

99. C'est moins vrai pour les peuples autochtones qui ont, dans de nombreux États, des mécanismes de représentation bien connus du droit interne.

100. Les populations autochtones sont liées par une "continuité historique avec les sociétés antérieures à l'invasion... et se jugent distinctes des autres éléments des sociétés qui dominent à présent sur leurs territoires", selon la définition du "rapport Cobo ", $c f$. p. 379 du chapitre XXI, site de l'ONU, voy. la série des $\mathrm{E} / \mathrm{CN} / 4 / \mathrm{sub}$.2. Toute définition doit prendre en compte l'auto-identification de l'individu à son groupe et l'acceptation de celui-ci par le groupe. La question de leur reconnaissance comme sujets directs du droit international semble bloquée. Mais, pour le propos qui est le nôtre, les mécanismes du droit interne qui leur reconnaissent une personnalité juridique sont largement suffisants. Les difficultés souvent avancées sur ce terrain pour leur refuser les droits intellectuels sont largement exagérées. Cf. P. RamDasi/S. Louafl, Protecting Traditional Knowledge, Genetic Resources And Folklore: The Way Forward, Les synthèses de l'IDDRI, n 8, 2006 ; pour le cas de la Nouvelle-Calédonie, M-A. HERMITTE, "La convention sur la diversité biologique et la question des droits intellectuels des peuples autochtones, une lacune française ", à paraître dans la Revue Juridique de l'Environnement, $n^{\circ}$ spécial sur le droit de l'environnement en Nouvelle-Calédonie. 
conduisent le prospecteur vers des ressources auxquelles elles accordent une importance particulière: plantes médicinales, rituelles, tinctoriales, poisons, etc. ; il se peut aussi que le prospecteur trouve des ressources auxquelles elles n'avaient pas prêté d'attention particulière. Dans les deux cas, il s'agit de ressources matérielles qui se trouvent sur le territoire qu'elles occupent et qu'elles ont a minima contribué à préserver par leur mode de vie. Dans le premier cas, outre les ressources, elles ont également fourni des connaissances, dites traditionnelles.

Or, le droit positif est ainsi fait que, sauf loi ou contrat organisant leurs droits, il ne confère à ces populations que peu de possibilités juridiques d'organiser la prospection en leur faveur. Elles vivent fréquemment sur des terres collectives, non délimitées autrement que par le droit coutumier et n'en maîtrisent pas l'accès. Leurs connaissances traditionnelles sont très souvent déjà divulguées par les récits de voyageurs, d'anthropologues ou, dans des pays comme la Chine et l'Inde, par des livres de médecine traditionnelle. À ce titre, elles font partie du domaine public, en libre accès pour tous. La reconnaissance internationale de leurs droits intellectuels permettrait de reconnaître plus facilement et de manière plus uniforme la contribution de leurs cultures, ce dont les représentants des peuples autochtones font maintenant une affaire de dignité. Or, il se trouve que la question a bien avancé dans les enceintes non dédiées à la propriété intellectuelle (3.1), alors que les organisations compétentes n'avancent que lentement, voire proposent des solutions plus dangereuses qu'utiles (3.2).

\subsection{Les enceintes internationales non dévolues à la propriété intellectuelle : des forces de proposition}

La question des droits intellectuels des peuples autochtones et des communautés locales a émergé dans des organisations internationales qui n'avaient pas de compétence en matière de propriété intellectuelle. Elles ont donc abordé le problème à partir de leurs logiques propres, celle du particularisme de la question du travail des autochtones pour l'OIT, celle du particularisme de leur approche des droits de l'homme pour l'ONU (a). La CDB s'est attachée à leur rôle dans la gestion de la diversité biologique (b).

\section{a. L'OIT et l'ONU : la découverte par l'Occident d'une vision autochtone du monde}

C'est en 1930 à l'OIT que les peuples autochtones apparaissent pour la première fois dans un texte juridique, cachés derrière la question du travail forcé imposé par les statuts de l'indigénat de la période coloniale. Amenée à réfléchir sur le travail, l'OIT fut la première à reconnaître les particularismes des peuples autochtones, de leurs valeurs culturelles et religieuses, du droit coutumier, de l'artisanat et des propriétés collectives ${ }^{101}$. L'OIT en arriva à conclure qu'ils ne pouvaient pas être purement et simplement absorbés par le droit commun, dans la perspective assimilationniste classique ; il y avait une place à trouver pour un ordre juridique spécifique.

101. Pour l'historique, $f f$. N. Rouland/S. PIERRÉ-CaPs/J. PoUmaréde : Droit des minorités et des peuples autochtones, PUF, 1996 et M.-A. HERMITTE (dir.), Les Ressources génétiques végétales et le droit dans les rapports nord-sud, Bruylant, Bruxelles, 2004. L'OIT avancera doucement, avec les conventions successives, 29 en 1930,107 en 1957 et 169 en 1989 
Le travail sera continué à l'ONU dans le cadre de la sous-commission des peuples autochtones ${ }^{102}$ qui s'attache à dessiner ce que pourrait être une autonomie partielle des peuples autochtones au sein des États, sous la forme d'un droit "d'intervenir dans les politiques qui les intéressent ". Non équivalente à la souveraineté, l'autonomie s'appuierait néanmoins sur des droits territoriaux, la reconnaissance de la culture, de la médecine, des langues, des activités traditionnelles, de la possibilité d'avoir une influence sur le système éducatif, etc.

Poursuivant dans cette voie, Irène Daes, rapporteur, a montré qu'il ne suffit pas d'aborder la valeur des cultures autochtones en tant que "patrimoines", mais qu'il faut les reconnaître comme pouvant être l'objet de droits intellectuels spécifiques. Elle part des atteintes qui leur ont été portées : sites sacrés bouleversés par l'industrie, l'agriculture et le tourisme, restes humains et objets sacrés captés à des fins scientifiques ou muséales, peintures traditionnelles détournées à des fins commerciales, etc. Elle pointe ensuite les difficultés liées à la défense juridique des cultures autochtones : échecs connus par les aborigènes d'Australie pour faire reconnaître le caractère collectif des droits sur les motifs traditionnels de peinture par les tribunaux, intérêt qu'il y aurait à certifier les produits artisanaux réalisés dans les réserves, etc. Elle fait remarquer que les catégories séparées du droit occidental, droits sur le patrimoine culturel, droits sur l'usage des terres, droits intellectuels, œuvres du domaine public, n'ont pas de sens dans la pensée autochtone qui lie entre eux des objets que le droit occidental disjoint : « la protection de la propriété culturelle et intellectuelle dépend pour l'essentiel de l'exercice des droits territoriaux et du droit des peuples autochtones à disposer d'eux-mêmes" ${ }^{103}$. Le patrimoine collectif comprend sans distinction les paysages, les espèces vivantes, les restes humains, les savoirs scientifiques, les objets d'art, tout ce qui participe du lien qui compose un peuple. Ce patrimoine peut être partagé mais pas aliéné, il n'est pas objet de propriété mais de responsabilité, le collectif en est le gardien (points 22 à 29). S'attaquant aux bioprospections, elle explique que les droits intellectuels occidentaux qui ne sont ni collectifs, ni perpétuels et sont tournés vers l'échange, sont incapables d'exprimer la vision autochtone du monde.

Tout ceci sera porté par le "groupe de travail des peuples autochtones " qui rédige un premier projet onusien de " déclaration des droits " en 1993 " 104 . Le texte se place d'emblée sur le fondement de l'égalité en dignité et en droits, "reconnaissant le droit de tous les peuples à être différents". C'est sur ce terrain que se situe la question des droits intellectuels : les cultures occidentales fonctionnent d'une certaine manière, et les propriétés intellectuelles portées par l'OMPI et l'OMC leur sont adaptées; les cultures autochtones sont autres, et ont besoin d'un système de droits intellectuels qui leur soit adapté. Dans sa version de novembre 2006, l'article 31 pose le principe d'une propriété intellectuelle collective applicable à tous les éléments du patrimoine naturel et culturel (savoir traditionnel, ressources humaines et génétiques, semences, pharmacopée, connaissance des propriétés de la faune et de la flore, traditions orales, etc.). Ils ont le droit de " préserver, contrôler, protéger et développer leur propriété intellectuelle collective de ce patrimoine culturel, ce savoir traditionnel [...]".

102. La commission des droits de l'homme a une sous-commission traitant de la protection des minorités, qui a elle-même une sous-commission sur les peuples autochtones. Cet empilement montre comment l'universalisme des droits de l'homme se décline sous des formes spécifiques.

103. E/CN.4/Sub.2/1993/28 du 28 juillet 1993 sur le site de l'ONU.

104. Elle avait été précédée de nombreuses tentatives, depuis la déclaration de 1981 de San José, la déclaration de Belem en 1988 rédigée par un congrès d'ethnobotanistes, la déclaration de Mataatua faite en 1993 à l'initiative de neuf tribus néo-zélandaises, etc. 
Le texte a été suspendu par le troisième comité de l'AG des Nations Unies devant l'opposition des grands États concernés, mais une partie de son contenu est reprise depuis plusieurs années sous un angle différent par les COP de la CDB.

b. La CDB et les « modes de vie traditionnels présentant un intérêt pour la diversité biologique".

C'est parce que certaines communautés ont un mode de vie préservant la diversité biologique ${ }^{105}$ qu'elles ont été mises en exergue par la CDB qui repose sur trois postulats: l'identité de régime juridique entre deux catégories différentes, les "communautés locales" et les "populations autochtones" 106 ; le souhait qu'elles entrent dans le «partage équitable des avantages "; le fait, enfin, qu'elles incarnent des " modes de vie traditionnels présentant un intërêt pour la conservation et l'utilisation durable de la diversité biologique " et qu'il est utile d'en favoriser " l'application sur une plus grande échelle ". L'État fournisseur est donc invité à élaborer une législation qui intègre ces postulats car la CDB part du principe de l'efficacité écologique des modes de vie traditionnels des communautés autochtones et locales, véritables sources d'inspiration pour l'avenir ${ }^{107}$.

La CDB inverse ainsi plusieurs siècles de perception des autochtones et de leurs savoirs. Simples traces du passé condamnées par l'histoire, ils deviennent modèles à suivre grâce à la crise écologique. Mais pour généraliser certaines de leurs pratiques, il faut y avoir accès et organiser juridiquement cette opération. Or, on l'a vu, le contrat est insuffisant car il dépend de la capacité à négocier une bonne figure contractuelle, ce qui est rarement à la portée des peuples autochtones, et moins encore des communautés locales. La création d'un droit intellectuel spécifique reconnu par la communauté internationale est donc la solution la plus efficace. C'est au fil des COP que les négociations ont pu aboutir à des "lignes directrices " qui apparaissent comme des étapes de la négociation dans l'espoir d'un texte à valeur obligatoire.

Adoptées en 2002, les "Lignes directrices de Bonn sur l'accès aux ressources génétiques et le partage juste et équitable des avantages résultant de leur utilisation ", portent au premier chef sur les rapports entre États fournisseurs et utilisateurs, mais donnent des indications sur les communautés autochtones et locales.

Les États fournisseurs doivent les informer et les consulter (XVI a et point 19), assurer leur participation au processus de négociation (point $14 \mathrm{~g} \mathrm{et} \mathrm{h}$ ), renforcer leurs capacités à être représentées et défendre leurs intérêts (point 16 a

105. Que l'affirmation soit vraie ou fausse n'entre pas en ligne de compte; elle a pour fonction de servir de fondement à un système d'attribution des droits. G. FilochE, Ethnodéveloppement, développement durable et droit en Amazonie, Bruxelles, Bruylant, 2007 : l'auteur, qui donne une précieuse étude de la situation en Amazonie, sous-estime les acquis et les possibilités futures d'évolution des textes internationaux, $c f$. particulièrement pp. 92 et $s$.

106. Autant les peuples autochtones ont fait l'objet de définitions précises même si elles ne sont pas forcément consensuelles, autant les communautés locales n'existent pas en droit et semblent renvoyer à des populations villageoises partageant des ressources et des savoirs autour de terroirs, de langues, de lignages, etc. La décision VI/10 de la COP note à son point 13 qu'il n'existe toujours aucune définition du concept de "communauté autochtone ou locale incarnant un mode de vie traditionnel ", ce qui devrait être pourtant examiné dans la tâche 12 du programme de travail.

107. Préambule, art. $8 \mathrm{j}$ et art. $10 \mathrm{c}$ de la CDB qui demande à chaque partie contractante de protéger et encourager "l'usage coutumier des ressources biologiques conformément aux pratiques culturelles traditionnelles compatibles avec les impératifs de leur conservation ou de leur utilisation durable ". 
VII), respecter les coutumes (point $16 \mathrm{~b}$ ii), veiller au partage des avantages, " conformément aux conditions convenues d'un commun accord avec les communautés" (point 16 b IX). Ce sont donc des conseils à la rédaction des contrats. Les utilisateurs devraient "encourager la divulgation de l'origine des connaissances, innovations et pratiques traditionnelles des communautés autochtones et locales dans les demandes de droits de propriété intellectuelle" (point $16 \mathrm{~d}$ ii) et mettre au point des "dispositifs de certification volontaire " de respect de ces règles (point $16 \mathrm{~d} \mathrm{~V}$ ), ce qui implique une intervention législative ${ }^{108}$. Si c'est à l'autorité nationale qu'il convient de demander l'autorisation d'accès à la ressource, les lignes directrices prévoient une participation des parties prenantes (point 19) et expriment le souhait que leur consentement soit obtenu, conformément au droit interne et aux pratiques coutumières (points $26 \mathrm{~d}$ et 31). Enfin, le partage des avantages devrait intégrer une part destinée aux communautés (point 48) ${ }^{109}$. Tout ceci constitue la trame de contrats plus qu'une solution générale, ce qui s'explique par la persistance de nombreuses oppositions ${ }^{110}$. En Europe, la Commission a réagi plutôt favorablement par une communication de 2003 qui entend préparer l'Union européenne à un avenir dans lequel la biopiraterie serait réglée par un texte obligatoire ${ }^{111}$. Elle s'attache essentiellement à ce que peuvent faire les États utilisateurs, les droits des communautés locales dépendant des décisions des États fournisseurs ${ }^{112}$. Ce n'est pas faux en soi mais lui permet "d'oublier "les peuples autochtones vivant sur le territoire européen, hormis une brève allusion au peuple Saami ${ }^{113}$. Du coup, la situation de la France d'outre-mer, par exemple, n'est pas évoquée. Bien plus, la Commission semble considérer qu'elle n'a aucune vocation à intervenir sur le sujet.

La septième COP a renforcé les droits des communautés locales, en liant le fondement de l'article $8 \mathrm{j}$ à celui de l'article 14 sur les études d'impact sur la diversité biologique. Conformément à la vision autochtone du monde qui ne sépare pas les éléments naturels et culturels, la COP a intégré aux impacts des projets d'aménagements sur la diversité biologique, leurs effets sur les communautés autochtones et locales. En 2004 elle a donc adopté les " Lignes directrices facultatives Akwé : Kon pour une conduite intégrée des études d'impact sur les sites sacrés, les terres et eaux occupées ou utilisées par les communautés autochtones et locales" ${ }^{114}$, ce qui comprend la bioprospection, "tout exercice de collecte [...]

108. À ce jour, six États membres de l'Union européenne l'ont intégré à leur législation.

109. [http://www.cbd.int/doc/publications/cbd-bonn-gdls-fr.pdf] ; UNEP/CBD/COP/6/20, p. 279, décision VI/24

110. Cf. le texte particulièrement négatif de l'industrie suisse, disponible sur le site de la CDB, UNEP/CDB/COP/4/Inf.16

111. Elle avait d'ailleurs été un contributeur important à la rédaction de ces lignes directrices, COM (2003) 821 final, SEC (2003) 1455, le 23 décembre 2003.

112. Sur ce point, cf. J-F. MORIN, “ La divulgation de l'origine des ressources génétiques, une contribution du droit des brevets à la protection de l'environnement ", in Droit et société, $\mathrm{n}^{\circ} 58,2004$, Ihttp://www.unisfera.org/IMG/pdf/Biodiversite_dans_regime_DPI. pdf]. L'une des pistes est le respect volontaire de codes d'éthique que se donnent différentes institutions, sur ce point, cf. C. NoIVILLE/ F. BELLIVIER, site internet de l'IDDRI, sur le code concernant les microorganismes [http:// bccm.belspo.be/projects/mosaicc/docs/code.pdfl; sur les systèmes de management environnemental et d'audit qui permettent aux entreprises de bénéficier d'une certification utilisée dans le marketing de l'entreprise, $c f$. Rgt.761/2001 du 19 mars 2001 permettant la participation volontaire des organisations à un système communautaire management environnemental et d'audit (EMAS), JOCE L114 du 24 avril 2001, p. 1 ; cf. aussi la recommandation 2001/680 du 7 septembre 2001, JOCE L247 du 17 septembre 2001 , p. 1 .

113. Il vit dans une vaste zone couvrant des portions des territoires de la Suède, la Norvège, la Finlande, la Sibérie et la Russie.

114. Décision VII/16, article $8 \mathrm{j}$ et dispositions connexes. 
devrait être effectué avec l'accord préalable en connaissance de cause des dépositaires de telles connaissances, innovations et pratiques [...]». Les lignes directrices prévoient une notification préalable de la prospection, avec identification des communautés potentiellement affectées et de leurs modalités de représentation, mise en place de mécanismes de participation, de recueil des opinions et préoccupations, procédure d'expression du consentement - on est toujours sur le modèle du contrat.

Mais les lignes directrices Akwé : Kon vont plus loin en affirmant que " le droit coutumier et les droits de propriété intellectuelle que les communautés autochtones et locales détiennent sur leurs connaissances [...] devraient être respectés. Ces connaissances ne devraient être utilisées qu'après obtention du consentement préalable en connaissance de cause de leurs propriétaires " (point V E 60). Elles s'attachent à « l'élaboration d'éléments pour des systèmes sui generis de protection des connaissances, innovations et pratiques traditionnelles" et comportent plusieurs points importants (point $\mathbf{H}$ ) : la préservation des connaissances est liée à la surveillance permanente par les communautés " des ressources biologiques situées sur des terres et des eaux " qu'elles occupent ou utilisent traditionnellement, ce qui rejoint cette conviction des communautés d'être "gardiennes " des plantes et des animaux. Le texte reconnaît ensuite que les communautés ont leurs propres systèmes coutumiers de protection de ces ressources et des connaissances qui y sont associées et pose les bases de leurs caractéristiques : répartition transfrontière, caractère collectif et inter-générationnel des ressources et des connaissances, importance des arrangements d'accès et de partage des avantages, intérêt d'un système sui generis des protections des connaissances traditionnelles à l'échelon international, sachant que ses mises en ouvre nationales devraient tenir compte du droit coutumier ( $c f$. aussi l'annexe des lignes directrices).

Ici encore, la position de l'Union européenne n'est pas claire, comme en témoigne le rapport de la Commission au Conseil sur « les progrès de la coopération avec les populations autochtones "115. D'un côté, la Commission développe des positions intéressantes sur le droit de celles-ci de "façonner leur propre développement social, économique et culturel et de modeler leur identité culturelle", les mécanismes d'intégration de la protection des autochtones dans l'évaluation des projets de développement et le principe de participation. Mais c'est applicable dans les rapports avec les pays d'Amérique latine, les projets réalisés au Botswana ou en Thaïlande, sans qu'une ligne soit consacrée à la politique européenne vis-à-vis des peuples autochtones européens, sans que rien ne vienne obliger les États membres à rendre des comptes sur leur politique nationale.

On peut donc constater que l'OIT, les Nations Unies et la CDB ont joué un rôle important comme enceintes de revendication et de négociation d'une base de compromis. En revanche, les États ont peu suivi, alors que la mise en cuvre de ces acquis dépend d'eux. En fait, la question des droits intellectuels sera sans doute tranchée, in fine, à l'OMPI avec ou sans l'appui de l'OMC et là, le paysage change.

\subsection{L'OMPI, instance d'arbitrage?}

Il est logique que les propositions de changement soient venues d'enceintes éloignées du droit de la propriété intellectuelle car elles ne sont pas bloquées par le poids de l'histoire des propriétés intellectuelles. Or, telles qu'elles existent aujourd'hui, les propriétés intellectuelles illustrent une vision du monde et de l'homme historiquement située, qui surinvestit la nouveauté des inventions et 
l'originalité des créations artistiques. Au centre se trouve l'homme des droits de l'homme, c'est-à-dire un individu exceptionnel pris dans la solitude de son universalité. Récompenser l'apport intellectuel de l'individu est une tâche sacrée que se donne le droit naturel ${ }^{116}$. Il en résulte un contrat social précis : aux inventeurs et créateurs qui apportent à l'humanité quelque chose de nouveau ou d'original, on octroie un monopole d'exploitation temporaire qui leur permet de tirer les fruits de leur apport. Une fois le délai expiré, l'invention et l'œuvre tombent dans le domaine public où tout un chacun peut en jouir et les exploiter. Patrimoine et œuvres sont séparés, comme nature et culture, corporel et incorporel, temps court du droit exclusif et temps long du domaine public.

Mais c'est une vision inversée du monde et de l'homme que proposent les civilisations autochtones. Ici, la transmission de la tradition prédomine et les évolutions s'y inscrivent discrètement ; les individus sont insérés dans des collectifs, le clan, la tribu, la lignée, l'aire linguistique. L'homme n'est pas séparé de la nature où il tisse de multiples liens avec les espèces vivantes et les paysages. Le contrat social occidental perd sa pertinence. Certes, l'idée de monopole existe sous la forme de l'exclusivité de l'autorité sur des espaces terrestres ou maritimes, sur des espèces totems, sur des dessins ou des chants. Il faut donc une autorisation pour y avoir accès, sans que le collectif qui a autorité en soit pour autant propriétaire. En revanche, c'est de tradition qu'il s'agit, même si elle est évolutive par certains aspects. Le caractère temporaire du monopole n'a pas de sens car les liens qui unissent les personnes et les choses sont imprescriptibles et inaliénables, comme la tradition. Les droits intellectuels des peuples autochtones s'expriment donc sous la forme du droit exclusif de donner l'accès aux ressources et aux connaissances. Ce droit est collectif, imprescriptible et inaliénable ; il empêche l'avènement d'un domaine public en libre accès, alors que celui-ci est au cœur du contrat social occidental. Cela n'empêche pas de multiples procédures de partage et d'échange sous condition de respect des procédures de consentement. Tant que le droit intellectuel occidental ne sera pas équilibré par son pendant autochtone, il manquera un élément essentiel à l'affirmation de l'égale dignité des civilisations.

Malgré son hétéronomie, le système s'articule sans difficulté avec le droit des contrats et le reste du droit de la propriété intellectuelle ; il est complémentaire et non contradictoire avec le droit commun, se situant en amont de la propriété intellectuelle classique et non en concurrence avec elle. Si l'on prend l'exemple du venin d'un animal sauvage intéressant en pharmacologie, l'accès au venin peut être autorisé à des conditions choisies par l'État fournisseur et les groupes concernés ; le travail de recherche effectué ensuite pourra donner lieu à brevet sans difficulté. Mais les communautés locales seront obligatoirement compensées au titre de leur propre propriété intellectuelle sur le venin. Pourtant, cela ne semble pas évident à l'OMPI. Pour le moment, dans la tradition occidentale, elle sépare la question des ressources génétiques de celle des connaissances traditionnelles. Se présentant comme le "cadre propice au débat ", elle cherche surtout les moyens d'éviter l'appropriation illicite des savoirs traditionnels et du folklore. C'est ainsi que depuis 1989, elle a créé un comité intergouvernemental sur la question, fait de multiples consultations avec les parties prenantes, y compris les peuples autochtones, élaboré des rapports et un projet sur le "patrimoine créatif " des peuples autochtones. Mais il s'agit de systèmes d'enregistrement de leurs connaissances et de leurs oeuvres, ce qui les divulgue ou consacre leur appartenance au domaine public où elles perdent leur valeur d'échange, ce qui

116. ...ce qui explique pourquoi le droit d'auteur est aux prises avec les formes de création contemporaine, instables et faisant intervenir de multiples acteurs. 
n'est pas le but recherché ${ }^{117}$. Pour le moment, l'OMPI semble incapable de concevoir un type de droit nouveau qui pourrait prendre modèle sur les appellations d'origine qui sont, elles-mêmes, collectives, imprescriptibles et inaliénables. Si les revendications sont bien mises sur la table des négociations, aucune décision, même partielle, n'a jamais été prise ${ }^{118}$.

$$
* *
$$

Le bilan de la CDB n'est pas nul, loin de là, mais son efficacité directe ne convainc pas. En fait, elle semblerait jouer surtout un rôle de proposition d'idées nouvelles, que les États, les régions, ou les autres organisations internationales choisissent d'adopter, ou non. L'auraient-ils fait si la convention ne les y avait pas incités? Probablement oui pour les parcs naturels terrestres, peut-être pour les parcs marins, sûrement pas pour la bioprospection, sûrement pas pour le système du consentement informé préalable aux importations d'organismes vivants modifiés. Certes, dans ces domaines, la mise en œuvre effective est encore rare, mais elle devrait croître avec le temps. Si l'utopie permet d'avancer, l'entêtement est nécessaire à la mise en œuvre, le commentateur devant, quant à lui, s'armer de patience.

117. M-A. HeRmitTe (dir.), Les Ressources génétiques végétales et le Droit dans les rapports nordsud, Bruylant, Bruxelles, 2004, pp. 87 et s.

118. Sur le travail à l'OMC, $c f$. les positions des États, plutôt fermées, sur le site de l'OMC, à la mention Domaines, propriétés intellectuelles, révision de l'article $273 \mathrm{~b}$ |http://www.wto.orgifrench/ tratop_f/trips_f/art27_3b_f. htm] ; le document IP/C/W/370/Rev. 1 en fait une synthèse au 9 mars 2006. 